\title{
Physiological and transcriptomic analyses reveal the mechanisms underlying the salt tolerance of Zoysia japonica Steud.
}

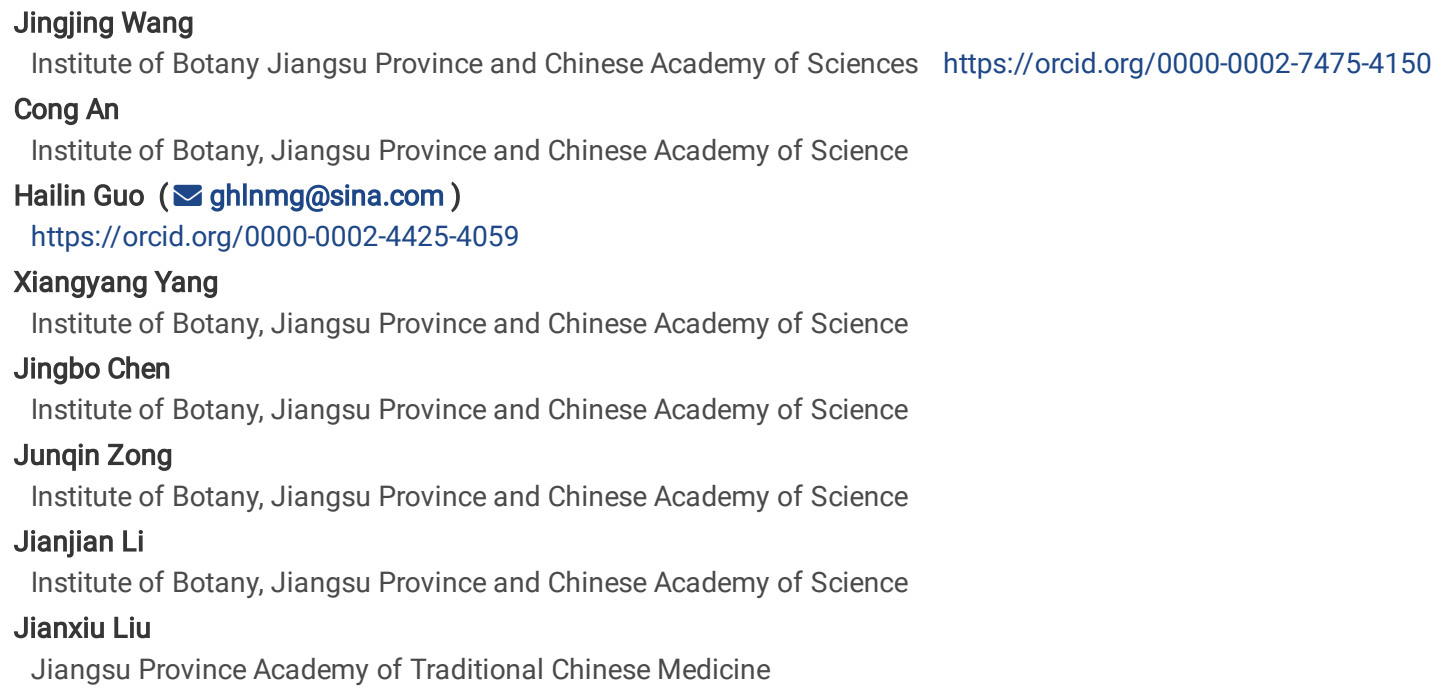

Version of Record: A version of this preprint was published at BMC Plant Biology on March 14th, 2020. See the published version at https://doi.org/10.1186/s12870-020-02330-6. 


\section{Abstract}

Soil salinization areas are sparsely populated and have fragile ecosystems, which seriously restricts the sustainable development of local economies. Zoysia grasses are recognized as excellent warm-season turfgrasses worldwide, with high salt tolerance and superior growth in saline-alkali soil. However, the mechanism underlying the salt tolerance of Zoysia remains unknown. In our study, we investigated the phenotypic and physiological responses of two contrasting materials, Zoysia japonica Steud. Z004 (salt sensitive) and Z011 (salt tolerant), to salt stress. The results showed that Z011 exhibited stronger salt tolerance than $\mathrm{Z004}$, with a higher $\mathrm{K}+/ \mathrm{Na}+$ ratio in both its leaves and roots. To further study the molecular mechanisms underlying salt tolerance, we compared the transcriptomes of the two materials at different time points ( $0 \mathrm{~h}, 1 \mathrm{~h}, 24 \mathrm{~h}$, and $72 \mathrm{~h})$ and from different tissues (leaves and roots) under salt treatment. The 24-h time point and roots were identified as the significant time point and tissue. According to the GO and KEGG analyses of different comparisons, the key DEGs participating in the salt-stress response were selected and belonged to the hormone pathway, TF families and the DUF family. The interaction between the key DEGs was discussed, revealing that auxin signal transduction and TF families may cooperate in Zoysia salt tolerance and that the WRKY family may be the most important TF family. Thus, our research provides fundamental information regarding the salt-stress response in Zoysia and enhances the understanding of molecular mechanisms in salt-tolerant plants.

\section{Background}

Soil salinization is a worldwide problem. Soil salinization areas are sparsely populated and have fragile ecosystems, which seriously restricts the sustainable development of local economies. As an important part of landscaping, turf plays an important role in protecting, improving and beautifying the urban environment. Therefore, it is particularly important to choose high-quality salt-tolerant turfgrass suitable for landscaping in soil salinization areas. Zoysia Willd. is a perennial herb belonging to the family Poaceae, subfamily Chloridoideae, tribe Zoysieae (Tsuruta et al., 2011). Zoysia grasses are recognized as excellent warm-season turfgrasses worldwide with salt tolerance, hardiness, and drought tolerance and are widely used in athletic fields, home lawns and parks (Ge et al., 2006). Compared to other Poaceae family members, Zoysia grasses have gained less attention in the research community. However, as an alternative grass species for landscaping in saline-alkali soil, Zoysia has superior growth qualities (Li et al., 2004). In particular, among the three most important commercial species, Zoysia japonica Steud. exhibits distinct tolerance to abiotic stress (Tsuruta et al., 2011). Therefore, it is of great significance to study the salt tolerance of Zoysia plants.

Early studies on salt tolerance in Zoysia mainly focused on the evaluation of salt tolerance and physiological mechanisms of salt tolerance. Salt tolerance evaluation results showed that the salt tolerance of Zoysia plants has rich genetic variation (Marcum et al., 1994; Qian et al., 2000; Weng and Chen, 2001; Li et al., 2012). It is convenient for us to select materials with contrasting salt tolerances for the study of the salt tolerance mechanism of Zoysia. Zoysia is a salt-secreting plant, and all of the Zoysia plant leaves have salt glands, which regulate the ion balance by selectively secreting saline ions. The salt tolerance of Zoysia plants is positively correlated with the rate of $\mathrm{Na}^{+}$secretion from salt glands in leaves and the density of salt glands per unit leaf area (Lee et al., 1994a; Lee et al., 1994b; Marcum et al., 1998). Previous studies have found that the salt tolerance of Zoysia is negatively correlated with the content of $\mathrm{Na}^{+}$and positively correlated with the content of $\mathrm{K}^{+}$in the leaf fluid. Salt-tolerant materials have a strong ability to maintain the $\mathrm{K}^{+} / \mathrm{Na}^{+}$content in leaves and roots. The content of $\mathrm{Na}^{+}$in leaves has been successfully applied to the evaluation of the salt tolerance of Zoysia (Marcum and Murdoch, 1990; Marcum et al., 1998; Li et al., 2012).

The salt tolerance of Zoysia is a very important trait, but to date, its molecular regulatory mechanism remains unknown. The Na+ $/ \mathrm{H}^{+}$antiporter gene $Z j N H X 1$, belonging to the plant NHX-gene family, was cloned from Z. japonica, and research results showed that ZjNHX1 plays an important role in ion homeostasis and salt tolerance (Du et al., 2010). The glycine-rich RNA-binding protein-coding gene ZjGRP was isolated from $Z$. japonica and was strongly induced by $\mathrm{NaCl}$ treatment. ZjGRP-overexpressing Arabidopsis thaliana plants exhibit low germination rates, seedling retardation and weakened salt tolerance (Teng et al., 2017). ZjZFN1 is a $\mathrm{C}_{2} \mathrm{H}_{2}$-type zinc finger protein-coding gene that is expressed higher in leaf tissues than in root and stem tissues and is induced by salt, cold and ABA treatments. Overexpressing ZjZFN1 in Arabidopsis thaliana can improve seed germination and enhance salt tolerance by improving the transcriptional activities of several salt-tolerance-related genes under salt stress (Teng et al., 2018).

Studies on the salt-tolerance genes in Zoysia remain rare. Chen et al. (2015) systematically excavated the salt-tolerance genes in Zoysia matrella using a full-length cDNA expression library in yeast, and 16 candidate salt-tolerance genes were identified, which are involved in ion regulation, osmotic adjustment, protein folding and modification, mitochondrial membrane translocase and RNA metabolism. Xie et al. (2015) presented the first comprehensive transcriptome data of Zoysia japonica Steud. roots, and a total of 32,849 unigenes and 4,842 SSRs were identified. This study shows that transcription factors (TFs) play significant roles in the early response to salt stress, including the AP2/EREBP family, bZIP family, NAC family, WRKY family, MYB family and $b H L H$ family (Xie et al., 2015).

Studies of the salt tolerance of zoysiagrass so far have focused on the evaluation of salt tolerance among different cultivars, the physiological mechanisms of salt tolerance and the development of molecular markers (Guo et al., 2014; Xu et al., 2015). However, the molecular mechanism of salt tolerance in zoysiagrass remains unclear. In this study, we investigated the phenotypic and physiological responses of two materials with contrasting salt tolerances, Z. japonica Z004 (salt sensitive) and Z011 (salt tolerant), to salt stress. Based on the existing Zoysia reference genome (Tanaka et al., 2016), the $\mathrm{HiSeq}^{\mathrm{TM}} 2000$ platform was used to perform RNA sequencing of zoysiagrass leaves and roots. We compared the transcriptomes of different time points (0 $\mathrm{h}, 1 \mathrm{~h}, 24 \mathrm{~h}$, and $72 \mathrm{~h}$ ) and different tissues (leaves and roots) under salt treatments to identify the significant time point and tissue. According to the GO and KEGG analysis of differentially expressed genes (DEGs) in different comparisons, the key DEGs participating in the salt-stress response were selected, and these DEGs belonged to the hormone pathway, TF families and the DUF family. The interaction between key DEGs was discussed, and these findings might reveal the gene network involved in Zoysia salt tolerance. Thus, our research provides fundamental information for use in future salt-stress studies of Zoysia and enhances the understanding of molecular mechanisms in salt-tolerant plants.

Page 2/18 


\section{Results}

\section{Phenotypic and physiological responses of $Z$. japonica Steud. to salt stress}

Japanese turfgrass (Zoysia japonica Steud.) is a popular and important warm-season turfgrass, and different accessions have different degrees of salt tolerance. In this study, two materials with contrasting salt tolerances, Z004 (salt sensitive) and Z011 (salt tolerant), were chosen for the analysis of the salttolerance mechanism in Z. japonica. The results of salt treatment showed that Z011 had strong salt tolerance with a good growth status, while Z004 was sensitive to salt and withered and yellowed after treatment with $350 \mathrm{mM} \mathrm{NaCl}$ for 40 days (Fig. 1a). Moreover, the leaf firing of Z004 was significantly higher than that of Z011 (Fig. 1b). The biomass statistics showed that the relative shoot clipping dry weight, verdure dry weight and root dry weight of Z011 were all markedly higher than those of Z004 (Fig. 1c-e).

To study the differences in the mechanism of salt tolerance between Z004 and Z011, the $\mathrm{Na}^{+}$and $\mathrm{K}^{+}$concentrations were detected in leaves, roots and secretions. In the control (CK), the $\mathrm{Na}^{+}$concentrations and $\mathrm{K}^{+}$concentrations in the leaves, roots and secretions were not significantly different between Z004 and Z011 (Fig. 2a-f). After treatment with $350 \mathrm{mM} \mathrm{NaCl}$, the $\mathrm{Na}^{+}$concentrations in the leaves, roots and secretions of Z004 and Z011 were all higher than those in the CK (Fig. 2a-c). In the roots of Z004 and Z011 after NaCl treatment, the $\mathrm{Na}^{+}$concentrations were not different (Fig. 2b). However, in the leaves, the $\mathrm{Na}^{+}$concentrations and secretions were significantly lower in Z011 than in Z004 (Fig. 2a, c). These results showed that Z011 may improve salt tolerance by reducing $\mathrm{Na}^{+}$absorption.

After treatment with $350 \mathrm{mM} \mathrm{NaCl}$, the $\mathrm{K}^{+}$concentrations in the leaves of Z004 and Z011 were lower than those in the leaves of the CK, but there were no differences in the $\mathrm{K}^{+}$concentrations between Z004 and Z011 (Fig. 2d). In addition, the $\mathrm{K}^{+}$concentrations in the roots of Z004 and Z011 were lower than those in the roots of the $\mathrm{CK}$, and the $\mathrm{K}^{+}$concentration in Z011 was significantly higher than that in Z004 (Fig. 2e). However, $\mathrm{K}^{+}$secretion in Z004 and Z011 after treatment with $\mathrm{NaCl}$ was higher than that in the $\mathrm{CK}$, and the $\mathrm{K}^{+}$secretion of Z004 was significantly higher than that of Z011 (Fig. $\mathbf{2 f}$ ). Therefore, compared with Z004, Z011 maintained a higher $\mathrm{K}^{+} / \mathrm{Na}^{+}$ratio in both the leaves and roots, which might be the reason for the stronger salt tolerance of Z011 (Fig. 2g, h).

\section{Transcriptome sequencing of the Z004 and Z011 accessions}

Leaf and root samples for RNA-seq were collected at 0 h, 1 h, 24 h and 72 h after treating Z004 and Z011 with 350 mM NaCl. In total, 16 samples were sequenced on the HiSeq ${ }^{\mathrm{TM}} 2000$ sequencing platform. We obtained an average of 28.8 million raw reads from the 16 libraries, and $97.18 \%$ of the sequences were confirmed as clean reads (Table 1). After that, the clean reads were mapped to the whole $Z$. japonica genome using Hisat2 (v2.0.5) software (Kim et al. 2015). A total of $62.7-91.39 \%$ of the total reads and $61.8-89.84 \%$ of the unique reads were mapped to the reference genome. In addition, multiple-mapped reads consisted of $0.85-1.67 \%$ of the total reads, and splice-mapped reads consisted of $17.58-32.42 \%$ of the total reads. Furthermore, $73.44-77.17 \%$ of the total reads were mapped to exons in the reference genome, $13.38-16.10 \%$ of reads were mapped to introns, and $8.58-12.06 \%$ of reads were mapped to intergenic regions (Online Resource 1). A total of 59,271 unigenes and 29,675 novel genes were revealed by RNA-seq assays. The number of unigenes $(62,172,69.90 \%)$ with lengths exceeding 1000 bp were more enriched than the unigenes $(21,909,24.63 \%)$ with lengths exceeding 300 bp and less than 1000 bp. For the convenience of comparison, we defined $0 \mathrm{~h}$ as the control group (CK) and $1 \mathrm{~h}, 24 \mathrm{~h}$ and $72 \mathrm{~h}$ as the treatment group (Tr). Principal component analysis (PCA) showed that the leaf and root samples of Z004 and Z011 were scattered in four different areas (Fig. 3a). Among them, the Z011 root samples had the highest dispersion degree (Fig. 3a). Moreover, the $24 \mathrm{~h}$ samples of the roots of Z004 and Z011 were separated from other samples, indicating that $24 \mathrm{~h}$ could be a crucial time (Fig. 3a).

Table 1 Summary of RNA-seq results and their matches to the $Z$. japonica genome

\begin{tabular}{|c|c|c|c|c|c|c|c|c|c|}
\hline $\mathbf{s}$ & $\begin{array}{c}\text { Raw } \\
\text { Reads }\end{array}$ & $\begin{array}{l}\text { Clean } \\
\text { Reads }\end{array}$ & $\begin{array}{l}\text { Clean } \\
\text { Bases }\end{array}$ & $\geq Q 30$ & $\begin{array}{c}\text { GC } \\
\text { Content }\end{array}$ & Mapped Reads & $\begin{array}{l}\text { Uniq Mapped } \\
\text { Reads }\end{array}$ & $\begin{array}{l}\text { Multiple Mapped } \\
\text { Reads }\end{array}$ & $\begin{array}{l}\text { Splice Mapped } \\
\text { Reads }\end{array}$ \\
\hline د & 32438255 & 31524956 & $9.46 \mathrm{G}$ & 94.6 & $55.83 \%$ & $57621250(91.39 \%)$ & $56624677(89.81 \%)$ & $996573(1.58 \%)$ & $18071152(28.66 \%)$ \\
\hline د & 26357459 & 25288464 & $7.59 \mathrm{G}$ & 92.5 & $54.76 \%$ & $45782098(90.52 \%)$ & $45017095(89.01 \%)$ & 765003(1.51\%) & 16292620(32.21\%) \\
\hline L & 24358613 & 23526320 & 7.06G & 92.71 & $54.56 \%$ & $42663657(90.67 \%)$ & $41993576(89.25 \%)$ & $670081(1.42 \%)$ & $12985607(27.6 \%)$ \\
\hline L & 31388773 & 30389440 & $9.12 \mathrm{G}$ & 94.74 & $55.81 \%$ & $55543678(91.39 \%)$ & $54603427(89.84 \%)$ & $940251(1.55 \%)$ & 18803898(30.94\%) \\
\hline$i$ & 28748978 & 27331853 & $8.2 \mathrm{G}$ & 94.85 & $56.39 \%$ & $45537682(83.31 \%)$ & $44882876(82.11 \%)$ & $654806(1.2 \%)$ & $14366719(26.28 \%)$ \\
\hline i & 25217276 & 24597846 & $7.38 \mathrm{G}$ & 92.33 & $54.40 \%$ & $30844803(62.7 \%)$ & $30401692(61.8 \%)$ & $443111(0.9 \%)$ & $10116388(20.56 \%)$ \\
\hline $\mathrm{R}$ & 32836307 & 31933917 & $9.58 \mathrm{G}$ & 94.73 & $54.30 \%$ & $48700816(76.25 \%)$ & $47999420(75.15 \%)$ & 701396(1.1\%) & 15287937(23.94\%) \\
\hline $\mathrm{R}$ & 36880428 & 35688473 & $10.71 \mathrm{G}$ & 94.71 & $54.97 \%$ & $56581667(79.27 \%)$ & $55714572(78.06 \%)$ & 867095(1.21\%) & $18346344(25.7 \%)$ \\
\hline 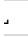 & 26586917 & 25922402 & $7.78 \mathrm{G}$ & 92.33 & $54.63 \%$ & $46443799(89.58 \%)$ & $45577309(87.91 \%)$ & $866490(1.67 \%)$ & $16809137(32.42 \%)$ \\
\hline 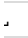 & 27176365 & 26429692 & 7.93G & 92.49 & $54.77 \%$ & $47016201(88.95 \%)$ & $46171840(87.35 \%)$ & $844361(1.6 \%)$ & $16310460(30.86 \%)$ \\
\hline L & 31282942 & 29168068 & $8.75 \mathrm{G}$ & 90.33 & $53.57 \%$ & $51780347(88.76 \%)$ & $50915346(87.28 \%)$ & $865001(1.48 \%)$ & $15892912(27.24 \%)$ \\
\hline L & 29732023 & 27463655 & $8.24 \mathrm{G}$ & 90.81 & $53.51 \%$ & 49171344(89.52\%) & 48301793(87.94\%) & 869551(1.58\%) & $16970162(30.9 \%)$ \\
\hline$l$ & 23872338 & 23011861 & $6.9 \mathrm{G}$ & 93 & $52.11 \%$ & $31773084(69.04 \%)$ & $31381515(68.19 \%)$ & $391569(0.85 \%)$ & $8092154(17.58 \%)$ \\
\hline$i$ & 31726696 & 30928060 & $9.28 \mathrm{G}$ & 90.35 & $54.36 \%$ & $45880277(74.17 \%)$ & $45225490(73.11 \%)$ & $654787(1.06 \%)$ & $14994212(24.24 \%)$ \\
\hline $\mathrm{R}$ & 20666337 & 20135617 & $6.04 \mathrm{G}$ & 91.57 & $56.20 \%$ & 30976008(76.92\%) & $30529687(75.81 \%)$ & $446321(1.11 \%)$ & $10196114(25.32 \%)$ \\
\hline $\mathrm{R}$ & 32232323 & 31023161 & $9.31 \mathrm{G}$ & 94.05 & $53.74 \%$ & $42725009(68.86 \%)$ & $42118724(67.88 \%)$ & $606285(0.98 \%)$ & $13160493(21.21 \%)$ \\
\hline
\end{tabular}




\section{Identification of DEGs in Z004 and Z011 in response to salt stress}

To characterize the differences between Z004 and Z011 in response to salt stress, we explored unigenes with significant expression level changes after $\mathrm{NaCl}$ treatment. The transcript abundance of each gene was calculated by fragments per kb per million fragments (FPKM) values. edgeR software (Robinson et al., 2010) was used to analyze significant differences in expression with padj $<0.05$ and fold change $>2$, and DEGs were identified with $\mid \log 2$ (Fold Change) $\mid>1$ and padj < 0.05. Under salt stress conditions, a total of 4,701 genes, 2,591 genes, 4,400 genes and 8,846 genes showed differential expression in Z004 leaves (Z0041L vs Z0040L, Z00424L vs Z0040L, Z00472L vs Z0040L), Z004 roots (Z0041R vs Z0040R, Z00424R vs Z0040R, Z00472R vs Z0040R), Z011 leaves (Z0111L vs Z0110L, Z01124L vs Z0110L, Z01172L vs Z0110L) and Z011 roots (Z0111R vs Z0110R, Z01124R vs Z0110R, Z01172R vs Z0110R), respectively. Interestingly, the number of DEGs in Z011 roots was 3.41 times that in Z004 roots, indicating that the difference in salt tolerance between Z004 and Z011 could be mainly in their roots. Moreover, in the comparisons of Z004 leaves, Z004 roots and Z011 leaves, the number of down-regulated genes was higher than the number of upregulated genes (Fig. 3b). However, in the comparisons of Z011 roots, the number of upregulated genes was more than the number of down-regulated genes (Fig. 3b). In addition, the number of DEGs in the 24-h sample (Z01124R vs Z0110R) was 2.28 and 5.64 times that in the 1-h (Z0111R vs Z0110R) and 72-h (Z01172R vs Z0110R) samples. The Venn diagrams showed that in the comparisons of Z004 roots, Z011 leaves and Z011 roots, the number of specific DEGs was higher at $24 \mathrm{~h}$ than at $1 \mathrm{~h}$ and $72 \mathrm{~h}$ (Supplementary Figure S1). These results indicated that $24 \mathrm{~h}$ could be a key time point for the salt-stress response.

\section{GO analysis of the DEGs in Z004 and Z011 roots}

For the functional characterization of DEGs, we assigned GO terms and selected significant GO classifications of DEGs in each comparison (padj < 0.05 ). In the Z0041R vs Z0040R comparison, 14 GO classes were identified, and all of them fell into the category 'molecular function'. In the Z00424R vs Z0040R comparison, $4 \mathrm{GO}$ classes fell into the category 'biological process', 1 fell into the category 'cellular component', and 20 fell into the category 'molecular function'. In the Z00472R vs Z0040R comparison, $2 \mathrm{GO}$ classes fell into the category 'biological process', 1 fell into the category 'cellular component', and 23 fell into the category 'molecular function'. In Z004R, 11 GO classes most relevant to the salt-stress response were concentrated in the Z00424R vs Z0040R comparison and Z00472R vs Z0040R comparison, including "response to oxidative stress", "response to stress", "extracellular region", "oxidoreductase activity, acting on paired donors, with incorporation or reduction of molecular oxygen", "oxidoreductase activity, acting on peroxide as acceptor", "antioxidant activity", "peroxidase activity", "iron ion binding", "ubiquitin-protein transferase activity", "ubiquitin-like protein transferase activity" and "sequence-specific DNA binding" (Online Resource 2). These results showed that Z004 responded slowly to salt stress. The significant stress response did not occur after $1 \mathrm{~h}$ of $\mathrm{NaCl}$ treatment but after $24 \mathrm{~h}$ and $72 \mathrm{~h}$.

In the Z0111R vs Z0110R comparison, $6 \mathrm{GO}$ classes fell into the category 'biological process', 16 fell into the category 'cellular component', and 10 fell into the category 'molecular function'. In the Z01124R vs Z0110R comparison, $10 \mathrm{GO}$ classes fell into the category 'biological process' and 5 fell into the category 'molecular function'. In the Z01172R vs Z0110R comparison, $2 \mathrm{GO}$ classes fell into the category 'biological process' and 8 fell into the category 'molecular function'. In Z011R, 5 GO classes most relevant to the salt-stress response were concentrated in its three comparisons, including "response to stress", "response to oxidative stress", "antioxidant activity", "peroxidase activity" and "oxidoreductase activity, acting on peroxide as acceptor" (Online Resource 2). These results showed that Z011 responded to salt stress faster than Z004. The stress response occurred after $1 \mathrm{~h}$ of $\mathrm{NaCl}$ treatment. By comparing the GO classes most relevant to salt stress in Z004R and Z011R, it was found that the 5 GO classes in Z011R coincided with Z004R. However, "extracellular region", "oxidoreductase activity, acting on paired donors, with incorporation or reduction of molecular oxygen", "iron ion binding", "ubiquitinprotein transferase activity", "ubiquitin-like protein transferase activity" and "sequence-specific DNA binding" were unique to Z004R and might be related to the differences in salt tolerance between Z004 and Z011.

\section{KEGG analysis of the DEGs in Z004 and Z011 roots}

The DEGs in Z004 and Z011 roots were mapped to KEGG pathways in Oryza sativa. In the Z0041R vs Z0040R, Z00424R vs Z0040R and Z00472R vs Z0040R comparisons, 40, 67 and 72 DEGs were assigned to the KEGG database involving 23, 40 and 32 pathways, respectively (Online Resource 3). In the Z0111R vs Z0110R, Z01124R vs Z0110R and Z01172R vs Z0110R comparisons, 100, 202 and 39 DEGs were assigned to the KEGG database involving 41, 52 and 26 pathways, respectively (Online Resource 3). The major pathways identified in the above comparisons were 'Metabolic pathways', 'Biosynthesis of secondary metabolites', 'Plant hormone signal transduction', 'MAPK signaling pathway - plant' and 'Phenylpropanoid biosynthesis'.

The statistical analysis of the number of DEGs in the above five pathways showed that most DEGs in Z004 were down-regulated, while most DEGs in Z011 were upregulated (Fig. 4). Especially in the MAPK signaling pathway, which is closely related to the plant response to abiotic stress, the DEGs in Z004 were all down-regulated, while in Z011, there were only 2 down-regulated DEGs, and all other DEGs were upregulated (Fig. 4d). In Z011, the number of DEGs was highest after $24 \mathrm{~h}$ of $\mathrm{NaCl}$ treatment, and the number of DEGs was lowest after $72 \mathrm{~h}$ (Fig. 4). In general, the number of DEGs involved in the salt-stress response in Z011 was significantly higher than that in Z004, and the number of upregulated genes obviously increased, indicating that the difference in salt tolerance between Z011 and Z004 might be mainly related to the upregulated genes in Z011 roots.

\section{Identification of DEGs from comparisons of Z004 and Z011 roots}

A total of 141 DEGs were identified with $\mid \log 2$ (Fold Change) $\mid \geq 2$ and padj $<0.05$ and selected from six different comparisons of Z004 and Z011 roots. In the hormone pathway, the results showed that the abscisic acid signal pathway had the most DEGs (17 genes), followed by the auxin signal pathway (13 genes) (Fig. 5a, Online Resource 4). The DEGs in the abscisic acid signal pathway all belonged to the protein phosphatase $2 C$ (PP2C) family, while in the 
auxin signal pathway, the 11 DEGs were related to auxin induction, and 2 DEGs were related to auxin response (Fig. 5a, Online Resource 4). In addition, 3 DEGs in the ethylene signal pathway belonged to the ethylene insensitive 3 (EIN3) family (Fig. 5a, Online Resource 4). In the selection of TFs, a total of 8 TF families were identified. The WRKYTF family had the most DEGs (30 genes), followed by the $b H L H$ TF family (19 genes) (Fig. 5 b, Online Resource 4). In addition, the bZIPTF family (10 genes), GRASTF family (10 genes), F-box TF family (7 genes), WD40 TF family (6 genes), SBPTF family (4 genes) and TCP TF family (3 genes) were identified (Fig. 5b, Online Resource 4). At the same time, a total of 14 genes in the DUF family, 4 ubiquitin genes and 1 heat-shock protein 70 (HSP70) were also identified (Fig. 5c, Online Resource 4).

From the above 141 DEGs, we selected 39 DEGs with significant inverse up- or downregulation in Z004 and Z011 roots after salt treatment (Table 2). Six DEGs in the hormone signal pathway were all down-regulated in Z004 roots and upregulated in Z011 roots. Among them, three DEGs belonged to the auxinresponsive protein small auxin-up RNA (SAUR) family in the auxin signal pathway, one DEG belonged to the EIN3 family in the ethylene signal pathway and two DEGs belonged to the PP2C family in the abscisic acid signal pathway. These six DEGs in Z011 roots all had the highest FPKM values at $24 \mathrm{~h}$ after salt treatment, which suggested that these six genes might have significant effects on salt tolerance (Table 2). In our research, members of various TF families involved in salt tolerance were identified. The major TF families presented were the WRKY and $b H L H$ families. Eleven $W R K Y$ and eight $b H L H$ transcription factors are listed in Table 2. All WRKYs were down-regulated in Z004 roots and upregulated in Z011 roots. Moreover, 7 bHLHTFs were also down-regulated in Z004 roots and upregulated in Z011 roots, except Zjn_sc00039.1.g03650.1.am.mk (bHLH30). In the bZIPTF family, five genes were selected, four of which were upregulated in Z004 roots and down-regulated in Z011 roots, except novel.1352 (bZIP53). In addition, three GRASTF family members, two WD40 TF family members and one SBPTF family member were all down-regulated in Z004 roots and upregulated in Z011 roots. A TCPTF family member, novel.30217 (TCP7), was upregulated in Z004 roots and down-regulated in Z011 roots (Table 2). Two DUF family members,

Zjn_sc00012.1.g05940.1.sm.mkhc (DUF315) and novel.26532 (DUF1671), were also selected and had an inverse expression trend, which might indicate that they have opposing functions (Table 2). From the above, 32 DEGs were down-regulated in Z004 roots and upregulated in Z011 roots, and 7 DEGs were upregulated in Z004 roots and down-regulated in Z011 roots, indicating that these genes might be related to the salt tolerance of Zoysia grass. Among them, the number of upregulated genes in the salt-tolerant variety Z011 roots were significantly greater than the number of down-regulated genes, indicating that these upregulated genes may play important roles in improving the salt tolerance of $Z$. japonica.

Table 2 Selected up- or down-regulated DEGs in Z004 and Z011 roots after salt treatments 


\begin{tabular}{|c|c|c|c|c|c|c|c|c|c|}
\hline \multirow[t]{2}{*}{ Gene ID } & \multirow[t]{2}{*}{ Gene family } & \multicolumn{3}{|c|}{ Z004R } & \multicolumn{3}{|c|}{ Z011R } & \multirow{2}{*}{$\begin{array}{l}\text { Up-down- } \\
\text { regulation }\end{array}$} & \multirow{2}{*}{$\begin{array}{c}\text { Putative } \\
\text { gene }\end{array}$} \\
\hline & & $\begin{array}{c}\log 2 \\
\text { Ratio(1h/0h) }\end{array}$ & $\begin{array}{c}\log 2 \\
\text { Ratio }(24 \mathrm{~h} / 0 \mathrm{~h})\end{array}$ & $\begin{array}{c}\log 2 \\
\text { Ratio }(72 \mathrm{~h} / 0 \mathrm{~h})\end{array}$ & $\begin{array}{c}\log 2 \\
\text { Ratio(1h/0h) }\end{array}$ & $\begin{array}{c}\log 2 \\
\text { Ratio }(24 \mathrm{~h} / 0 \mathrm{~h})\end{array}$ & $\begin{array}{c}\log 2 \\
\text { Ratio }(72 \mathrm{~h} / 0 \mathrm{~h})\end{array}$ & & \\
\hline s00020.1.g02750.1.sm.mk & Auxin_inducible & -0.72 & -2.76 & -0.94 & 1.68 & 4.41 & 1.86 & down/up & SAUR41 \\
\hline 200002.1.g13200.1.sm.mk & Auxin_inducible & -2.86 & -3.15 & -3.63 & 1.85 & 2.49 & -0.51 & down/up & SAUR77 \\
\hline 200003.1.g11120.1.sm.mk & Auxin_inducible & -1.69 & -2.80 & -2.55 & 3.45 & 4.16 & 0.66 & down/up & SAUR77 \\
\hline z00001.1.g00520.1.sm.mk & EIN3 & -1.91 & -2.15 & -1.73 & 0.99 & 2.26 & 0.16 & down/up & EIL1 \\
\hline novel.25564 & PP2C & -0.19 & -1.68 & -0.98 & 2.66 & 3.43 & 1.28 & down/up & PP2C12 \\
\hline novel.30342 & PP2C & -0.12 & -0.64 & -0.56 & 1.41 & 2.41 & 1.02 & down/up & PP2C27 \\
\hline j0069.1.g00340.1.sm.mkhc & WRKY & -1.61 & -0.78 & -2.17 & 1.92 & 1.20 & -0.80 & down/up & WRKY28 \\
\hline novel.1285 & WRKY & -0.19 & -1.00 & -0.50 & 0.54 & 2.13 & 0.31 & down/up & WRKY35 \\
\hline novel.72 & WRKY & -1.27 & -1.58 & -2.36 & 0.06 & 0.60 & -1.28 & down/up & WRKY38 \\
\hline 200009.1.g08230.1.sm.mk & WRKY & -4.40 & -2.45 & -5.91 & 3.10 & 2.78 & -0.09 & down/up & WRKY38 \\
\hline j0086.1.g02720.1.sm.mkhc & WRKY & -2.15 & -2.00 & -2.30 & 2.04 & 2.03 & -0.46 & down/up & WRKY40 \\
\hline s00011.1.g02540.1.sm.mk & WRKY & -0.65 & -1.33 & -1.47 & 1.79 & 2.07 & 0.29 & down/up & WRKY56 \\
\hline J0040.1.g03610.1.am.mkhc & WRKY & -2.95 & -3.06 & -3.47 & 4.10 & 5.09 & 1.39 & down/up & WRKY64 \\
\hline j0011.1.g02350.1.sm.mkhc & WRKY & -0.51 & -1.54 & -1.18 & 1.97 & 3.96 & 0.83 & down/up & WRKY65 \\
\hline j0029.1.g04940.1.sm.mkhc & WRKY & 0.01 & -0.77 & -0.31 & 0.88 & 3.33 & 0.45 & down/up & WRKY65 \\
\hline novel.23742 & WRKY & -3.69 & -1.83 & -3.70 & 2.15 & 1.05 & -0.18 & down/up & WRKY70 \\
\hline J0045.1.g02590.1.am.mkhc & WRKY & -2.35 & -1.76 & -2.26 & 1.98 & 1.84 & -0.35 & down/up & WRKY71 \\
\hline s00011.1.g01200.1.sm.mk & bHLH & -2.07 & -1.44 & -2.59 & 2.20 & 0.75 & -0.35 & down/up & bHLH13 \\
\hline 200029.1.g03690.1.sm.mk & bHLH & -5.19 & -2.55 & -4.24 & 1.90 & 0.39 & -1.20 & down/up & bHLH13 \\
\hline 200039.1.g03650.1.am.mk & bHLH & -0.15 & 0.77 & 0.20 & -0.96 & -2.23 & -0.17 & up/down & bHLH30 \\
\hline 200005.1.g03230.1.am.mk & bHLH & -0.87 & -2.83 & -2.21 & 1.09 & 4.64 & -0.28 & down/up & bHLH41 \\
\hline 200008.1.g06800.1.am.mk & bHLH & -1.45 & -1.86 & -2.56 & 2.01 & 3.63 & 0.09 & down/up & bHLH41 \\
\hline novel.1977 & bHLH & -1.83 & -1.89 & -2.45 & 2.12 & -1.12 & -0.73 & down/up & bHLH94 \\
\hline 200032.1.g00520.1.am.mk & bHLH & -2.67 & -1.32 & -2.65 & 1.18 & 0.64 & -1.18 & down/up & bHLH96 \\
\hline novel.8275 & bHLH & 0.17 & -0.90 & 0.71 & 0.35 & 2.41 & 1.92 & down/up & bHLH101 \\
\hline novel.4508 & bZIP & 0.47 & 0.63 & 0.76 & -1.88 & -2.63 & -0.56 & up/down & TGAL6 \\
\hline 200018.1.g02790.1.am.mk & bZIP & -0.28 & 0.42 & -0.42 & -2.09 & -1.67 & -1.08 & up/down & bZIP2 \\
\hline 200037.1.g05090.1.sm.mk & bZIP & 0.81 & 1.21 & 0.92 & -1.61 & -2.47 & -1.25 & up/down & bZIP11 \\
\hline s00045.1.g04610.1.sm.mk & bZIP & -0.07 & 1.05 & 0.27 & -0.68 & -2.28 & -0.72 & up/down & bZIP11 \\
\hline novel.1352 & bZIP & -0.51 & -2.22 & -0.30 & 0.42 & 1.55 & 0.68 & down/up & bZIP53 \\
\hline novel.24172 & GRAS & -0.70 & -0.99 & -0.49 & 1.56 & 3.49 & 1.52 & down/up & SCL9 \\
\hline novel.8767 & GRAS & 0.07 & -0.81 & 0.29 & 2.09 & 4.33 & 2.01 & down/up & SCL9 \\
\hline 200103.1.g00280.1.am.mk & GRAS & -0.31 & -1.55 & 0.12 & 1.19 & 2.54 & 1.72 & down/up & NSP1 \\
\hline novel.3563 & WD40 & -0.53 & -1.03 & -0.77 & 1.47 & 3.17 & 1.09 & down/up & WDR44 \\
\hline j0027.1.g02480.1.sm.mkhc & WD40 & -0.39 & -1.40 & -0.75 & 1.81 & 3.87 & 1.10 & down/up & RACK1A \\
\hline novel.32257 & SBP & -0.15 & -0.81 & -0.36 & 1.52 & 2.20 & 0.45 & down/up & SPL15 \\
\hline novel.30217 & TCP & 0.32 & 0.83 & 0.57 & -1.55 & -2.66 & -1.01 & up/down & TCP7 \\
\hline 00012.1.g05940.1.sm.mkhc & DUF315 & 0.54 & 0.96 & 0.20 & -0.98 & -2.72 & -1.33 & up/down & ROPGEF7 \\
\hline novel.26532 & DUF1671 & -0.29 & -0.33 & -0.40 & 1.83 & 2.58 & 1.32 & down/up & UFSP \\
\hline
\end{tabular}

\section{Verification of transcriptome sequencing data of Z004 and Z011 roots}

To verify the reliability of the transcriptome sequencing data of Z004 and Z011 roots, twenty of the 39 DEGs with significant inverse up- or downregulation in Z004 and Z011 roots after salt treatment were selected and validated using quantitative real-time PCR (qRT-PCR). Approximately $90.0 \%$ of the DEGs were consistent between the RNA-seq and qRT-PCR data, with the exception of Zjn_sc00029.1.g04940.1.sm.mkhcand Zjn_sc00011.1.g02540.1.sm.mk (Supplementary Figure S2). The results of the qRT-PCR and RNA-seq data were generally consistent, indicating that our transcriptome sequencing data of Z004 and Z011 roots were reliable.

\section{Discussion}

\section{Phenotypic and physiological responses to salt tolerance in Z. japonica Steud.}

Zoysia is a warm-season turfgrass that is now widely used in home lawns, football fields and ecological management (Teng et al., 2017). Zoysia grass is recognized for its salt tolerance, hardiness, and drought tolerance and is suitable as a high-quality salt-tolerant turfgrass for landscaping in soil salinization areas (Ge et al., 2006). Previous studies have found that the salt tolerance of Zoysia is negatively correlated with the content of $\mathrm{Na}^{+}$and positively correlated with the content of $\mathrm{K}^{+}$in leaf fluids. Salt-tolerant materials have a strong ability to maintain the $\mathrm{K}^{+} / \mathrm{Na}^{+}$content in leaves and roots (Marcum and Murdoch, 1990; Marcum et al., 1998; Li et al., 2012). Our data for two materials with contrasting salt tolerances support these previous studies (saltsensitive material Z004 and salt-tolerant material Z011). After $350 \mathrm{mM} \mathrm{NaCl}$ treatment for 40 days, Z011 exhibited stronger salt tolerance than Z004, with a good growth status and higher biomass (Fig. 1a-e). Compared with Z004, Z011 maintained $\mathrm{Na}^{+}$concentrations that were significantly lower in leaves and secretions, but there was no differences in roots (Fig. 2a-c). Moreover, there was no difference in the $\mathrm{K}^{+}$concentration in the leaves of $\mathrm{Z011}$ compared with the leaves of Z004, but the $\mathrm{K}^{+}$concentration was significantly higher in the roots and lower in the secretions of Z011 than in Z004 (Fig. $\mathbf{2 d - f ) . ~ T h e s e ~ r e s u l t s ~}$ 
indicated that Z011 may improve salt tolerance by reducing $\mathrm{Na}^{+}$absorption, enhancing $\mathrm{K}^{+}$absorption and reducing $\mathrm{K}^{+}$secretion to maintain a significantly higher $\mathrm{K}^{+} / \mathrm{Na}^{+}$ratio in both its leaves and roots (Fig. $\mathbf{2 g}, \mathbf{h}$ ).

\section{Transcriptome sequencing and DEG identification in Z004 and Z011 in response to salt stress}

Xie et al. (2015) presented the first comprehensive transcriptome data of Zoysia japonica Steud. roots after $30 \mathrm{~min}$ of $\mathrm{NaCl}$ treatment, and a total of 32,849 unigenes and 4,842 SSRs were identified. However, the organs of the roots and leaves that had more important regulatory capacities under salt stress and the key time points for salt tolerance regulation remain unknown. Our research sampled the leaves and roots from Z004 and Z011 at $0 \mathrm{~h}, 1 \mathrm{~h}, 24 \mathrm{~h}$ and $72 \mathrm{~h}$ after treatment with $350 \mathrm{mM} \mathrm{NaCl}$, and a total of 59,271 unigenes and 29,675 novel genes were revealed by RNA-seq assays. PCA analysis showed that the Z011 root samples had the highest dispersion degree, and the 24-h samples of the roots of Z004 and Z011 were separated from other samples (Fig. 3a). Moreover, the number of DEGs in Z011 roots was 3.41 times that in Z004 roots, and the number of DEGs at $24 \mathrm{~h}$ (Z01124R vs Z0110R) was 2.28 and 5.64 times that at $1 \mathrm{~h}$ (Z0111R vs Z0110R) and $72 \mathrm{~h}$ (Z01172R vs Z0110R) (Fig. 3b, Supplementary Figure S1). These results indicated that the difference in salt tolerance between $\mathrm{Z} 004$ and Z011 could be mainly in their roots, and $24 \mathrm{~h}$ could be a key time point for the salt-stress response.

\section{Plant hormone signal transduction is involved in salt tolerance}

Further evidence in the KEGG analysis of the DEGs in Z004 and Z011 roots demonstrated that plant hormone signal transduction has important effects on salt tolerance (Fig. 4c). Six DEGs, which might have significant effects on salt tolerance, were selected for plant hormone signal transduction and were all down-regulated in Z004 roots and upregulated in Z011 roots. Among them, one SAUR41 and two SAUR77 genes belonged to the auxin-responsive protein SAUR family in the auxin signal pathway, one ethylene-insensitive-3-like-1 (EIL 1) gene belonged to the EIN3 family in the ethylene signal pathway and one PP2C12 and one PP2C27 gene belonged to the PP2C family in the abscisic acid signal pathway (Table 2).

SAURs are a family of auxin-responsive genes that play an important role in the regulation of plant growth and development. However, the function of the SAUR family in salt tolerance has rarely been reported. Previous studies have only shown that TaSAUR75 was down-regulated in wheat roots after salt stress, and higher root length, survival rate and expression of some salt and drought stress-responsive genes were detected in TaSAUR75-overexpressing transgenic lines than in control plants (Guo et al., 2018). In Arabidopsis, the overexpression of AtSAUR41 promoted hypocotyl elongation and increased primary root growth and lateral root numbers (Kong et al., 2013). Yeast two-hybrid experiments showed that AtSAUR77 might participate in ethylene receptor signaling and promote plant growth (Li et al., 2015). The function of these two genes in salt tolerance has not yet been reported. In our research, under salt tolerance, both ZmSAUR41 and ZmSAUR77 were down-regulated in Z004 roots and upregulated in Z011 roots, indicating that ZmSAUR41 and ZmSAUR77 might improve salt tolerance (Table 2).

Ethylene signaling has been regarded as a stress hormone involved in the plant response to salt tolerance. EIN3 and EIL 1 are two ethylene-activated transcription factors and have been reported to be important for enhancing salt tolerance. In Arabidopsis, the accumulation of EIN3/EIL1 was induced by high salinity and likely enhanced reactive oxygen species (ROS) scavenging at the seedling stage to promote salinity tolerance because the ein3 eil 1 double mutant had higher levels of ROS, lower levels of peroxidase-encoding transcripts and hypersensitivity to salt tolerance (Peng et al., 2014). However, in contrast, the overexpression OSEIL 1 and OsEIL 2 in rice resulted in salt hypersensitivity at the seedling stage, and the lack of OsEIL 1 and OsEIL2 functions enhanced salt tolerance. Furthermore, the negative regulation of OSEIL 1 and OSEIL2 in salt tolerance might contribute to the regulation of high-affinity $K^{+}$ transporter 2;1 expression and the absorption of $\mathrm{Na}^{+}$in roots (Yang et al., 2015). In our research, under salt tolerance, ZmEIL 1 was down-regulated in Z004 roots and upregulated in Z011 roots, indicating that ZmEIL 1 might have a positive effect on salt tolerance, consistent with AtEIL 1 in Arabidopsis (Table 2).

PP2C enzymes are key elements in the ABA signaling pathway. In the present study, $P P 2 C$ family genes had different expression levels in response to salt tolerance. Overexpressing AtPP2CG1 (Arabidopsis thaliana protein phosphatase 2C G Group 1) in Arabidopsis could enhance salt tolerance, whereas a lack of AtPP2CG1 function caused decreased salt tolerance. In addition, AtPP2CG1 upregulated some stress-responsive gene expression levels under salt treatment, including RD29A, RD29B, DREB2A and KIN1 (Liu et al., 2012). In maize, salt treatment induced intron methylation of ZmPP2C and then caused the expression of $Z m P P 2 C$ to be significantly down-regulated (Tan, 2010). Transcriptomic analysis of Camellia sinensis revealed that multiple $P P 2 C$ members participated in the salt-tolerance response, such as $P P 2 C 2, P P 2 C 3, P P 2 C 14, P P 2 C 51$ and $P P 2 C 60$, which were upregulated under salt treatments, but PP2C12, PP2C27 and PP2C54 were down-regulated (Wan et al., 2018). In our study, for salt tolerance, both ZmPP2C12 and ZmPP2C27 were downregulated in Z004 roots and upregulated in Z011 roots, indicating that ZmPP2C12 and ZmPP2C27 might be positive regulators of salt tolerance (Table 2).

\section{Transcription factors involved in salt tolerance}

In our research, various TF families involved in salt tolerance were identified. Among them, the members of the $W R K Y$ and $b H L H T F$ families were the most common (Table 2). The WRKYTF family is known to be involved in various physiological processes and many aspects of the plant defense system. We identified eleven differentially expressed WRKY genes, and seven of them were reported to be related to salt tolerance. In Gossypium raimondii, Cai et al. (2014) found that WRKY35, WRKY40 and WRKY64 were induced by salt, and the expression levels were significantly upregulated. Moreover, AtWRKY40 was found to be induced in response to salt stress in Arabidopsis (Seki et al., 2002). Overexpressing FcWRKY40 of Fortunella crassifolia in tobacco and lemon enhanced salt tolerance, while silencing FCWRKY40 decreased salt tolerance (Dai et al., 2018). Wang et al. (2019) cloned the WRKY56 gene from Populus simonii $\times$ Populus nigra, which was significantly induced by salt treatment and transformed it into Arabidopsis. WRKY56-overexpressing transgenic Arabidopsis plants exhibited stronger salt tolerance compared with wild type, including an increase in fresh weight and germination (Wang et al., 2019). A PsnWRKY70 gene was also cloned from P. simonii $\times$ P. nigra and confirmed the response to salt stress in PsnWRKY70-repressed plants, which showed enhanced salt tolerance (Zhao et al., 2017). In addition, AtWRKY70 has been reported to regulate salt stress by interacting with the Cys2/His2 zinc finger protein Zat7 (Ciftci-Yilmaz et al., 2007). WRKY71 and WRKY28 were homologs and induced by high salinity. The overexpression of WRKY71 or WRKY28 
resulted in insensitive flowering of Arabidopsis to high salinity, while the decline in WRKY71 and WRKY28 resulted in more sensitive flowering of Arabidopsis (Yu et al., 2017). Given that the transcripts of WRKYs in our research were all down-regulated in Z004 roots and upregulated in Z011 roots, the $W R K Y$ genes may play positive roles in salt tolerance.

The $b H L H T F$ family has been reported to participate in regulating abiotic stress-related signal transduction. Eight differentially expressed $b H L H$ genes were identified in our research, and four of them have been reported to be regulated by $\mathrm{NaCl}$ (Table 2). The $b H L H 30$ genes in chrysanthemum, evergreen tree and upland cotton were all induced under salt stress and indicated to enhance salt tolerance in yeast (Chen et al., 2018; Wang et al., 2016; Shi et al., 2015). In Arabidopsis, two presumptive paralogs of $b H L H 92, b H L H 41$ and $b H L H 42$, were induced by salt treatment (Jiang et al., 2009). Moreover, in the transcriptome analysis of salinity tolerance in Brassica juncea, bHLH101 showed downregulation under salt treatments (Sharma et al., 2015). In our research, the $b H L H 13$, bHLH41, bHLH94, bHLH96 and bHLH101 genes were down-regulated in Z004 roots and upregulated in Z011 roots, indicating that these $b H L H$ genes might be positive regulators of salt tolerance. Among them, the expression trend of bHLH101 in Zoysia was in contrast to that in Brassica juncea (Sharma et al., 2015), indicating that $b H L H 101$ may have different functions in different species. In addition, $b H L H 30$ might play a negative role in salt tolerance in Zoysia, with upregulation in Z004 roots and downregulation in Z011 roots, which contrasts with previous reports.

The $b Z I P T F$ family has important roles in many biological processes, and some $b Z I P T F$ family members have been reported to have biological functions under salt stress. Transgenic plants overexpressing AtbZIP1, ZmbZIP72, GmbZIP132 and ZmABP9 exhibited enhanced tolerance to salt stress (Sun et al., 2012; Ying et al., 2012; Liao et al., 2008; Wang et al., 2017). Five differentially expressed bZIP genes were identified in our research, and $b Z I P 2$ and $b Z I P 53$ were reported to be involved in salt tolerance (Table 2). The transcript level of the LebZIP2 gene in Lycopersicon esculentum was increased after salt-stress treatments, and the bZIP1 and bZIP53 mutants showed reduced salt tolerance (Seong et al., 2008; Hartmann et al., 2015). In our research, TGAL6, bZIP2 and bZIP11 were all upregulated in Z004 roots and down-regulated in Z011 roots, while bZIP53 was down-regulated in Z004 roots and upregulated in Z011 roots, indicating that different bZIP members might play different roles in the salt tolerance process in $Z$. japonica.

Among the remaining seven transcription factors (Table 2), only the salt tolerance function of the $R A C K 1 A$ gene had been clearly reported. In rice, the suppression of OSRACK1A enhanced salt tolerance by maintaining low $\mathrm{Na}^{+}$and high $\mathrm{K}^{+}$concentrations in both roots and leaves (Zhang et al., 2018). Our data showed that three GRASTF family members (SCL9 and NSP1), two WD40 TF family members (WDR44 and RACK1A) and one SBPTF family member (SPL15) were all down-regulated in Z004 roots and upregulated in Z011 roots, while a TCPTF family member (TCP7) was upregulated in Z004 roots and down-regulated in $\mathrm{Z} 011$ roots. These genes may have important roles in salt tolerance, but their functions require further research.

\section{DUF family is involved in salt tolerance in Z. japonica}

DUF family members consist of a large number of uncharacterized protein families in the Pfam database (http://pfam.xfam.org/family), which contains approximately 3000 families (Bateman et al., 2010). Some DUF-containing proteins are active in plant development (Bischoff et al., 2010; Cao et al., 2010), and other members of DUF families are involved in the stress response (Kim et al., 2012; Wang et al., 2014), especially in the salt-stress response. The AhDGR2 gene in Amaranthus hypochondriacus encodes a DUF642 protein, and plants overexpressing AhDGR2 had increased sensitivity to NaCl treatment (Palmeros-Suárez et al., 2016). OsDSR2, encoding a DUF966 domain protein, also negatively regulates salt stress in rice (Luo et al., 2014). However, overexpressing SIDP361 (a DUF1644 protein gene) in rice significantly enhanced salt tolerance at the seedling and heading stages (Li et al., 2016). The expression of OSDUF810.7 was significantly increased under salt treatment, and the overexpression of this gene in $E$. coli improved the salt resistance of bacteria (Li et al., 2018). Two DUF family members, ROPGEF7 (a DUF 315 protein-coding gene) and UFSP (a DUF 1671 protein-coding gene), were selected in our research and showed an inverse expression trend (Table 2), indicating that these two DUF genes might play important roles in the salt tolerance process in $Z$. japonica and have contrasting functions.

\section{The predicted interaction clusters of significant DEGs under salt stress}

The salt tolerance response is a complex process that relies on interactions of different stress-responsive genes. Hence, the interaction analysis of 39 significant DEGs in Table 2 was performed in STRING (https://string-db.org/), and three interaction clusters were observed (Fig. 6). To obtain more information regarding these interactions, we chose Arabidopsis thaliana as a reference species. In cluster I, SAUR77 interacted with SAUR41 and bHLH94, while bHLH94 interacted with WRKY70. WRKY70 interacted with bHLH96, WRKY40, and WRKY38 and indirectly interacted with WRKY28 and TCP7. These results imply that auxin signal transduction, the $b H L H T F$ family, the WRKYTF family and the TCPTF family may cooperate in the salt tolerance response of Zoysia. In cluster II, WRKY56 interacted with bHLH30 and indirectly interacted with bHLH41. Moreover, bHLH41 interacted with bZIP53, bZIP53 interacted with RACK1A and bZIP11 and indirectly interacted with bZIP2. These results imply that the WRKYTF family, the $b H L H T F$ family, the $b Z I P T F$ family and the WD40 TF family may cooperate in the salt tolerance response of Zoysia. In cluster III, WRKY71 indirectly interacted with WRKY65, and WRKY65 indirectly interacted with WRKY35. Considering these interactions, auxin signal transduction and TF families may cooperate in Zoysia salt tolerance, and the WRKY family may be the most important TF family in salt-stress regulation.

\section{Methods}

\section{Plant materials and treatment}

Based on salt tolerance identification of 206 zoysiagrass accessions using Leaf firing, the relative shoot clipping dry weight, verdure dry weight and root dry weight (H. L. Guo, unpublished data), the salt sensitive Z. japonica accession Z004 and the salt tolerant Z. japonica accission Z011 were used in this study. Both accessions were collected in 1995, Z004 was collected in Lushan area (Jiangxi, China) and Z011 was collected in Lanxi area (Zhejiang, China), and maintained at an experimental field of the Institute of Botany, Jiangsu Province and Chinese Academy of Sciences. Previous morphological and DNA 
analysis confirmed the correct identfication of the two accessions (Liu et al., 2005; Guo et al., 2008). Twenty uniform sprigs of two materials with contrasting salt tolerances, Z004 and Z011, from the experimental field of the Institute of Botany were planted into $9 \mathrm{~cm}$-diameter and $6 \mathrm{~cm}$-deep plastic pots filled with coarse silica sand. Ten pots were planted for each treatment and each material. Pots were suspended over tanks $\left(66.56 \times 45.56 \times 17.0 \mathrm{~cm}{ }^{3}\right)$ filled with $45 \mathrm{~L} 1 / 2$ Hoagland's solution. Grasses were clipped weekly until growth was consistent. The grasses were cultivated in a greenhouse with a day/night temperature of $35 / 28{ }^{\circ} \mathrm{C}, 16 \mathrm{~h}$ of light/ $8 \mathrm{~h}$ of dark, $75 \%$ relative humidity and $800 \mu \mathrm{mol} \mathrm{m}^{-2} \mathrm{~s}^{-1}$ photosynthetically active radiation. NaCl treatment (350 $\mathrm{mM})$ was initiated after two months of cultivation.

\section{Observation and measurement of salt tolerance in Z. japonica Steud.}

After exposure to the salt treatment for 40 days, the leaf firing of grasses was assessed by visual rates using a scale of 1 (slight firing) to 9 (severe firing). All treatments and control grasses were divided into three parts, including shoot clipping, verdure and root. Among them, the part of the grasses clipped to a height of $4.0 \mathrm{~cm}$ belonged to the shoot clipping, and the part excluding the shoot clipping and root belonged to verdure. These three parts were dried at 70 ${ }^{\circ} \mathrm{C}$ for $48 \mathrm{~h}$, and the dry weight was determined. The relative dry weights were calculated according to the following equation: Relative dry weight = $\mathrm{Wt} / \mathrm{Wo} \times 100 \%$, where Wt is the dry weight in $\mathrm{NaCl}$ treatments, and Wo is the dry weight in control. Three biological replicates were performed, and data from individual measurements were averaged and analyzed using SPSS Statistics v. 18.0 ( $t$ test) (SPSS Inc., Chicago, IL, USA).

\section{Determination of the concentration of $\mathrm{Na}^{+}$and $\mathrm{K}^{+}$}

To determine the concentrations of $\mathrm{Na}^{+}$and $\mathrm{K}^{+}$in the leaves and roots, $20 \mathrm{mg}$ of dry powder samples was placed into sealed test tubes containing $15 \mathrm{ml}$ $\mathrm{ddH}_{2} \mathrm{O}$. All samples were boiled for $1 \mathrm{~h}$ and kept at room temperature for $24 \mathrm{~h}$. After filtering, the extracted $\mathrm{Na}^{+}$and $\mathrm{K}^{+}$concentrations were measured by flame photometry (Model FP6410; Shanghai Xinyi Instruments Inc., Shanghai, China) and calculated on the basis of the tissue dry weight (mmol kg ${ }^{-1}$ ). To determine the secretion of $\mathrm{Na}^{+}$and $\mathrm{K}^{+}$, the leaves were washed with $\mathrm{ddH}_{2} \mathrm{O} 3$ times after 5 days of salt treatment to remove the salt crystals secreted from the leaf surface. Seven days later, 4-8 pieces of mature leaves were taken and placed into centrifuge tubes. Each centrifuge tube was supplemented with 10

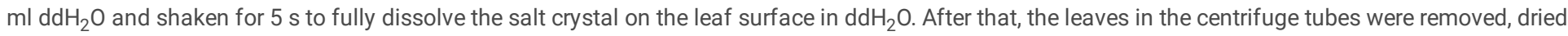
at $70{ }^{\circ} \mathrm{C}$ for $48 \mathrm{~h}$, and the dry weight was weighed. The $\mathrm{Na}^{+}$and $\mathrm{K}^{+}$concentrations in $10 \mathrm{ml} \mathrm{ddH}_{2} \mathrm{O}$ were measured by flame photometry and calculated on the basis of the corresponding leaf dry weight $\left(\mathrm{mmol} \mathrm{kg}^{-1}\right)$. The $\mathrm{K}^{+} / \mathrm{Na}^{+}$ratio is the ratio of the $\mathrm{K}^{+}$concentration to the $\mathrm{Na}^{+}$concentration. Three biological replicates were performed, and data from individual measurements were averaged and analyzed using SPSS Statistics v. 18.0 ( $t$ test) (SPSS Inc., Chicago, IL, USA).

\section{RNA-seq}

The leaf and root tissues of Z004 and Z011 at four time points ( 0 h, 1 h, 24 h, and $72 \mathrm{~h})$ after salt treatment were sampled, frozen in liquid nitrogen and stored at $-80^{\circ} \mathrm{C}$. Each collected sample was $0.1 \mathrm{~g}$, and three biological replicates were performed. Total RNA was extracted (Xie et al., 2015), and every three biological replicate RNA samples were mixed fully into one RNA library. A total of 16 RNA libraries were generated after the samples were pooled, including Z0040L (Z004, 0 h, leaf), Z0041L (Z004, 1 h, leaf), Z00424L (Z004, 24 h, leaf), Z00472L (Z004, 72 h, leaf), Z0040R (Z004, 0 h, root), Z0041R (Z004, 1 h, root), Z00424R (Z004, 24 h, root), Z00472R (Z004, 72 h, root), Z0110L (Z011, 0 h, leaf), Z0111L (Z011, 1 h, leaf), Z01124L (Z011, 24 h, leaf), Z01172L (Z011, 72 h, leaf), Z0110R (Z011, 0 h, root), Z0111R (Z011, 1 h, root), Z01124R (Z011, 24 h, root), and Z01172R (Z011, 72 h, root). The cDNA libraries and transcriptome sequencing were performed at the Novogene (Tianjin, China) (http://www.novogene.com/) on an Illumina HiSeq ${ }^{\mathrm{TM}} 2000$ platform, and 125 bp/150 bp paired-end reads were generated. The datasets are available in the NCBI repository http://www.ncbi.nlm.nih.gov/bioproject/PRJNA559944.

\section{De novo assembly, gene expression level and identification of DEGs}

Clean reads were obtained from raw data by removing reads containing adapters, reads with ambiguous bases ' $N$ ' and low-quality reads. The Q20, Q30 and GC content of the clean reads were calculated, and the downstream analyses were based on the high-quality clean reads. De novo assembly of the $Z$. japonica transcriptome in the reference genome of zoysiagrasses (Tanaka et al., 2016) was accomplished using Hisat2 (v2.0.5) (Kim et al., 2015). The mapped reads of each sample were assembled, and the novel transcripts were predicted by StringTie (v1.3.3b) (Pertea et al., 2015). The gene expression levels were calculated using the FPKM method (Mortazavi et al., 2008). The edgeR R package (3.18.1) (Robinson et al., 2010) was used to analyze significant differences in expression with padj $<0.05$ and fold change $>2$, and DEGs were identified with $\mid \log 2$ (Fold Change) $\mid>1$ and padj $<0.05$.

\section{GO and KEGG enrichment analysis of DEGs}

All DEGs were mapped to each term of the GO and KEGG (http://www.genome.jp/kegg/) databases, and the clusterProfiler R package was used to analyze GO enrichment of DEGs and test the statistical enrichment of the DEGs in KEGG pathways. The GO terms and KEGG categories with padj < 0.05 (corrected P-value) were selected as significant GO classifications and KEGG pathways for the DEGs in each comparison.

\section{qRT-PCR validation of transcription}

Twenty DEGs were selected from the 39 DEGs with significant inverse up- or downregulation in Z004 and Z011 roots after salt treatment to validate the reliability of the transcriptome data. The primers of DEGs were designed using Primer 5.0 software, and ZjActin (GenBank: GU290545.1) was used as a housekeeping gene. Each sample had three biological replicates, and qRT-PCR assays were carried out as described by Xie et al. (2015). The gene primers are listed in Online Resource 5. 


\section{Abbreviations}

cDNA: Complementary DNA; CK: Control group; DEGs:differentially expressed genes; FPKM: Fragments per kb per million fragments; GO: Gene ontology; KEGG: Kyoto Encyclopedia of Genes and Genomes; NCBI: National Center for Biotechnology Information; PCA: Principal component analysis; qRT-PCR: Quantitative real-time PCR; RNA-seq: RNA sequence; TF: Transcription factors; Tr: The treatment group.

\section{Declarations}

\section{Acknowledgments}

Not applicable.

\section{Authors' contributions}

HG, XY, JC and JZ performed the physiological experiments. JW, CA and JJL performed the transcriptomic analyses and verification experiments. HG designed the experiment. JXL supervised the project. JW and HG participated in writing the manuscript. All authors read and approved the final manuscript.

\section{Funding}

This work was funded by the National Natural Science Foundation of China [31572155, 31672195], and the Natural Science Foundation of Jiangsu Province, China [BK20151371].

\section{Availability of data and materials}

The datasets analyzed during the current study are available from the corresponding author on reasonable request. All data generated or analyzed during this study are included in this published article [and its Additional files].

\section{Ethics approval and consent to participate}

Not applicable.

\section{Consent for publication}

Not applicable.

\section{Competing interest}

The authors declare that they have no conflict of interest.

\section{References}

Bateman A, Coggill P, Finn RD (2010) DUFs: families in search of function. Acta Crystallogr Sect F Struct Biol Cryst Commun. 66(10), 1148-1152. doi: $10.1107 / \mathrm{S} 1744309110001685$

Bischoff V, Nita S, Neumetzler L, Schindelasch D, Urbain A, Eshed R, Persson S, Delmer D, Scheible W (2010) TRICHOME BIREFRINGENCE and Its Homolog AT5G01360 Encode Plant-Specific DUF231 Proteins Required for Cellulose Biosynthesis in Arabidopsis. Plant Physiol 153(2), 590-602. doi: $10.1104 /$ pp. 110.153320

Cai CP, Niu E, Du H, Zhao L, Feng Y, Guo WZ (2014) Genome-wide analysis of the WRKY transcription factor gene family in Gossypium raimondii and the expression of orthologs in cultivated tetraploid cotton. Crop J 2(2), 87-101. doi: 10.1016/j.cj.2014.03.001

Cao X, Yang KZ, Xia C, Zhang XQ, Chen LQ, Ye D (2010) Characterization of DUF724 gene family in Arabidopsis thaliana. Plant Mol Biol 61-73. doi: $10.1007 / \mathrm{s} 11103-009-9551-5$

Chen Y, Zong JQ, Tan ZQ, Li LL, Hu BY, Chen CM, Chen JB, Liu JX (2015) Systematic mining of salt-tolerant genes in halophyte-zoysia matrella through cdna expression library screening. Plant Physiol Bioch 89, 44-52. doi: 10.1016/j.plaphy.2015.02.007

Cheng PL, Gao JJ, Feng YT, Zhang ZX, Liu YN, Fang WM, Chen SM, Chen FD, Jiang JF (2018) The chrysanthemum leaf and root transcript profiling in response to salinity stress. Gene 161-169. doi: 10.1016/j.gene.2018.06.075

Ciftciyilmaz S, Morsy MR, Song L, Coutu A, Krizek BA, Lewis MW, Warren D, Cushman J, Connolly EL, Mittler R (2007) The EAR-motif of the Cys2/His2-type Zinc Finger Protein Zat7 Plays a Key Role in the Defense Response of Arabidopsis to Salinity Stress. J Biol Chem 282(12), 9260-9268. doi: 10.1074/jbc.M611093200

Dai WS, Wang M, Gong XQ, Liu JH (2018) The transcription factor FcWRKY40 of Fortunella crassifolia functions positively in salt tolerance through modulation of ion homeostasis and proline biosynthesis by directly regulating SOS2 and P5CS1 homologs. New Phytol 219(3):972-989. doi: $10.1111 /$ nph.15240 
Du YH, Hei Q, Liu YX, Zhang H, Xu K, Xia T (2010) Isolation and Characterization of a Putative Vacuolar Na ${ }^{+} \mathrm{H}^{+}$Antiporter Gene from Zoysia japonica L. J Plant Biol 53: 251-258. doi: 10.1007/s12374-010-9107-x

Ge YX, Norton T, Wang ZY (2006) Transgenic zoysiagrass (Zoysia japonica) plants obtained by Agrobacterium-mediated transformation. Plant Cell Rep 25, 792-798. doi: 10.1007/s00299-006-0123-8

Guo HL, Ding WW, Chen JB, Chen X, Zheng YQ, Wang ZY, Liu JX (2014) Genetic linkage map construction and QTL mapping of salt tolerance traits in Zoysiagrass (Zoysia japonica). PLoS One 9:e107249. doi: 10.1371/journal.pone.0107249

Guo HL, Liu JX, Zhou ZF, Xuan JP. Interspecific relationship and genetic diversity of zoysiagrass revealed by SSR markers. Acta Agrestia Sinica, 2008, 16(6): 552-557.Guo Y, Jiang QY, Hu Z, Sun XJ, Fan SJ, Zhang H (2018) Function of the auxin-responsive gene TaSAUR75 under salt and drought stress. Crop J 181-190. doi: 10.1016/j.cj.2017.08.005

Hartmann LH, Pedrotti L, Weiste C, Fekete A, Schierstaedt J, Gottler J, Kempa S, Krischke M, Dietrich K, Mueller MJ, Carbajosa JV, Hanson J, Dröge-Laser W (2015) Crosstalk between Two bZIP Signaling Pathways Orchestrates Salt-Induced Metabolic Reprogramming in Arabidopsis Roots. Plant Cell 27(8), 22442260. doi: $10.1105 /$ tpc. 15.00163

Jiang YQ, Yang B, Deyholos MK (2009) Functional characterization of the Arabidopsis bHLH92 transcription factor in abiotic stress. Mol Genet Genomics 282(5), 503-516. doi: 10.1007/s00438-009-0481-3

Kim D, Langmead B, Salzberg SL (2015) Hisat: a fast spliced aligner with low memory requirements. Nat Methods 12(4), 357-360. doi: 10.1038/nmeth.3317

Kim SJ, Ryu MY, Kim WT (2012) Suppression of Arabidopsis RING-DUF1117 E3 ubiquitin ligases, AtRDUF1 and AtRDUF2, reduces tolerance to ABA mediated drought stress. Biochem. Biophys. Res. Commu 420, 141-147. doi: 10.1016/j.bbrc.2012.02.131

Kong YY, Zhu YB, Gao C, She WJ, Lin WQ, Chen Y, Han N, Bian HW, Zhu MY, Wang JH (2013) Tissue-specific expression of SMALL AUXIN UP RNA41 differentially regulates cell expansion and root meristem patterning in Arabidopsis. Plant Cell Physiol 54, 609-621. doi: 10.1093/pcp/pct028

Lee GJ, Yoo YK, Kim KS (1994a) Comparative salt tolerance study in Zoysiagrasses \. Interspecific comparison among eight zoysiagrasses (Zoysia spp.). J. Kor. Soc. Hort. Sci. 35(2): 178-185.

Lee GJ, Yoo YK, Kim KS (1994b) Comparative Salt Tolerance Study in Zoysiagrasses III. Changes in Inorganic Constituents and Proline Contents in Eight Zoysiagrasses (Zoysia spp.). J. Kor. Soc. Hort. Sci. 35(3): 241-250.

Li LH, Lv MM, Li X, Ye TZ, He X, Rong SH, Dong YL, Guan Y, Gao XL, Zhu JQ, Xu ZJ (2018) The Rice OsDUF810 Family: OsDUF810.7 may be involved in the tolerance to salt and drought. Mol Biol 52(4), 489-496. doi: 10.1134/S002689331804012X

Li M, Guo LJ, Guo CM, Wang LJ, Chen L (2016) Over-expression of a DUF1644 protein gene, SIDP361, enhances tolerance to salt stress in transgenic rice. J Plant Biol 59(1), 62-73. doi: 10.1007/s12374-016-0180-7

Li S, Chen JB, Guo HL, Zong JQ, Zhang F, Chu XQ, Jiang QF, Ding WW, Liu JX (2012) Salinity tolerance evaluation of Zoysia turfgrass germplasm. Acta Prataculturae Sin 21(4), 43-51.

Li Y, Geng L, Liu JX (2004) Assessment on salt-tolerance of Zoysia spp. in China. Acta Agrestia Sin 12, 8-11. doi: 10.1088/1009-0630/6/5/011

Li ZG, Chen HW, Li QT, Tao JJ, Bian XH, Ma B, Zhang WK, Chen SY, Zhang J (2015) Three SAUR proteins SAUR76, SAUR77 and SAUR78 promote plant growth in Arabidopsis. Sci. Rep 5, 12477. doi: 10.1038/srep12477

Liao Y, Zhang J, Chen SY, Zhang WK (2008) Role of Soybean GmbZIP132 under abscisic acid and salt stresses. J Integr Plant Biol 50(2), 221-230. doi: 10.1111/j.1744-7909.2007.00593.x

Liu JX, Guo HL, Zhu XH, Gao H, Sun ZY. The comprehensive assessment on the germplasm of zoysia spp. Acta Agrestia Sinica, 2005, $13(3)$ : 219-256.

Liu X, Zhu YM, Zhai H, Cai H, Ji W, Luo X, Li J, Bai X (2012) AtPP2CG1, a protein phosphatase 2C, positively regulates salt tolerance of Arabidopsis in abscisic acid-dependent manner. Biochem Bioph Res Co 422(4). doi: 10.1016/j.bbrc.2012.05.064

Luo CK, Guo CM, Wang WJ, Wang LJ, Chen L (2014) Overexpression of a new stress-repressive gene OsDSR2, encoding a protein with a DUF966 domain increases salt and simulated drought stress sensitivities and reduces aba sensitivity in rice. Plant Cell Rep 33(2), 323-336. doi: 10.1007/s00299-013-1532-0

Marcum KB, Anderson SJ, Engelke MC (1998) Salt gland ion secretion: A salinity tolerance mechanism among five zoysiagrass species. Crop Sci 38: 806810. doi: 10.2135/cropsci1998.0011183X003800030031x

Marcum KB, Murdoch CL (1990) Growth responses, ion relations, and osmotic adaptations of eleven C4 turfgrasses to salinity. Agron J 82: 892-896. doi: 10.2134/agronj1990.00021962008200050009x 
Marcum KB, Murdoch CL (1994) Salinity Tolerance Mechanisms of Six C4 Turfgrasses. J. AMER. Soc. Hort. Sci 119(4): 779-784. doi: 10.21273/jashs.119.4.779

Mortazavi A, Williams BA, Mccue K, Schaeffer L, Wold BJ (2008) Mapping and quantifying mammalian transcriptomes by RNA-Seq. Nat Methods 5(7):621628. doi: $10.1038 /$ nmeth.1226

Palmeros-Suárez PA, Massange-Sánchez JA, Sánchez-Segura L, Martínez-Gallardo NA, Espitia RE, Gómez-Leyva JF, Délano-Frier JP (2016) AhDGR2, an amaranth abiotic stress-induced DUF642 protein gene, modifies cell wall structure and composition and causes salt and ABA hyper-sensibility in transgenic Arabidopsis. Planta 245(3), 1-18. doi: 10.1007/s00425-016-2635-y

Peng JY, Li ZH, Wen X, Li WZ, Shi H, Yang LS, Zhu HQ, Guo HW (2014) Salt-induced stabilization of EIN3/EIL1 confers salinity tolerance by deterring ROS accumulation in Arabidopsis. PLoS Genet 10(10), e1004664. doi: 10.1371/journal.pgen.1004664

Pertea M, Pertea G, Antonescu CM, Chang TC, Mendell JT, Salzberg SL (2015) StringTie enables improved reconstruction of a transcriptome from RNA-seq reads. Nat Biotechnol 33(3), 290-295. doi: 10.1038/nbt.3122

Qian YL, Engelke MC, Foster MJV (2000) Salinity effects on zoysiagrass cultivars and experimental lines. Crop Sci 40: 488-492. doi: 10.2135/cropsci2000.402488x

Robinson MD, McCarthy DJ, Smyth GK (2010) edgeR: a Bioconductor package for differential expression analysis of digital gene expression data. Bioinformatics 26(1): 139-140. doi: 10.1093/bioinformatics/btp616

Seki M, Narusaka M, Ishida J, Nanjo T, Fujita M, Oono Y, Kamiya A, Nakajima M, Enju A, Sakurai T, Satou M, Akiyama K, Taji T, Yamaguchi-Shinozaki K, Carninci P, Kawai J, Hayashizaki Y, Shinozaki K (2002) Monitoring the expression profiles of 7000 Arabidopsis genes under drought, cold and high-salinity stresses using a full-length cDNA microarray. Plant J 31(3), 14. doi: 10.1046/j.1365-313X.2002.01359.x

Seong ES, Kwon SS, Ghimire BK, Yu CY, Cho DH, Lim JD, Kim KS, Heo K, Lim ES, Chung IM, Kim MJ, Lee YS (2008) LebZIP2 induced by salt and drought stress and transient overexpression by Agrobacterium. J Biochem Mol Biol 41(10), 693-698. doi: 10.5483/BMBRep.2008.41.10.693

Sharma R, Mishra M, Gupta BK, Parsania C, Singlapareek SL, Pareek A (2015) De novo assembly and characterization of stress transcriptome in a salinitytolerant variety CS52 of Brassica juncea. PLOS ONE 10(5). doi: 10.1371/journal.pone.0126783

Shi GY, Guo XY, Guo JY, Liu LH, Hua JP (2015) Analyzing serial cDNA libraries revealed reactive oxygen species and gibberellins signaling pathways in the salt response of Upland cotton (Gossypium hirsutum L.). Plant Cell Rep 34(6), 1005-1023. doi: 10.1007/s00299-015-1761-5

Sun XL, Li Y, Cai H, Bai X, Ji W, Ding XD, Zhu YM (2012) The Arabidopsis AtbZIP1 transcription factor is a positive regulator of plant tolerance to salt, osmotic and drought stresses. J Plant Res 125(3), 429-438. doi: 10.1007/s10265-011-0448-4

Tan MP (2010) Analysis of DNA methylation of maize in response to osmotic and salt stress based on methylation-sensitive amplified polymorphism. Plant Physiol Bioch 48(1), 21-26. doi: 10.1016/j.plaphy.2009.10.005

Tanaka H, Hirakawa H, Kosugi S, Nakayama S, Ono A, Watanabe A, Hashiguchi M, Gondo T, Ishigaki G, Muguerza M, Shimizu K, Sawamura N, Inoue T, Shigeki Y, Ohno N, Tabata S (2016) Sequencing and comparative analyses of the genomes of zoysiagrasses. DNA Res 23(2), 171-180. doi:

10.1093/dnares/dsw006

Teng K, Tan PH, Guo WE, Yue YS, Fan XF, Wu JY (2018) Heterologous expression of a novel Zoysia japonica C2H2 zinc finger gene, ZjZFN1, improved salt tolerance in Arabidopsis. Front Plant Sci 9, 1159. doi: 10.3389/fpls.2018.01159

Teng K, Tan PH, Xiao GZ, Han LB, Chang ZH, Chao YH (2017) Heterologous expression of a novel Zoysia japonica salt-induced glycine-rich RNA-binding protein gene, ZjGRP, caused salt sensitivity in Arabidopsis. Plant Cell Rep 36, 179-191. doi: 10.1007/s00299-016-2068-x

Tsuruta SI, Kobayashi M, Ebina M (2011) "Zoysia," In: kole C. (eds) Wild Crop Relatives: Genomic and Breeding Resources. (Berlin; Heidelberg: Springer) 297-309.

Wan SQ, Wang WD, Zhou TS, Zhang YH, Chen JF, Xiao B, Yang YJ, Yu YB (2018) Transcriptomic analysis reveals the molecular mechanisms of Camellia sinensis in response to salt stress. Plant Growth Regul 84(1), 1-12. doi: 10.1007/s10725-017-0354-4

Wang CL, Lu GQ, Hao YQ, Guo HM, Guo Y, Zhao J, Cheng HM (2017) ABP9, a maize bZIP transcription factor, enhances tolerance to salt and drought in transgenic cotton. Planta 246(3), 453-469. doi: 10.1007/s00425-017-2704-x

Wang L, Shen RX, Chen LT, Liu YG (2014) Characterization of a novel DUF1618 gene family in rice. J Integr Plant Biol 56(2), 151-158. doi: $10.1111 /$ jipb. 12130

Wang L, Yao WJ, Sun Y, Wang JY, Jiang TB (2019) Association of transcription factor WRKY56 gene from Populus simoniixP. nigra with salt tolerance in Arabidopsis thaliana. PeerJ 7:e7291. doi: 10.7717/peerj.7291 
Wang XH, Gao BW, Liu X, Dong XJ, Zhang ZX, Fan HY, Zhang L, Wang J, Shi S, Tu PF (2016) Salinity stress induces the production of 2-(2-phenylethyl) chromones and regulates novel classes of responsive genes involved in signal transduction in Aquilaria sinensis calli. BMC Plant Biol 16, 1. doi: $10.1186 / s 12870-016-0803-7$

Weng JH, Chen YC (2001) Variation of Salinity Tolerance in Zoysia Clones Collected from Different Habitats in Taiwan. Plant Prod. Sci 4(4): 313-316. doi: $10.1626 / p p s .4 .313$

Xie Q, Niu J, Xu XL, Xu LX, Zhang YB, Fan B, Liang XH, Zhang LJ, Yin SX, Han LB (2015) De novo assembly of the Japanese lawngrass (Zoysia japonica Steud.) root transcriptome and identification of candidate unigenes related to early responses under salt stress. Front Plant Sci 6 . doi: $10.3389 /$ fpls. 2015.00610

Xu LX, Zhang ML, Zhang XZ, Han LB (2015) Cold acclimation treatment-induced changes in abscisic acid, cytokinin, and antioxidant metabolism in Zoysiagrass (Zoysia japonica). HortScience 50, 1075-1080. doi: 10.1007/s10142-011-0213-8

Yang C, Ma B, He SJ, Xiong Q, Duan KX, Yin CC, Chen H, Lu X, Chen SY, Zhang JS (2015) Maohuzi6/ethylene insensitive3-like1 and ethylene insensitive3like2 regulate ethylene response of roots and coleoptiles and negatively affect salt tolerance in rice. Plant Physiol 169(1), 148-65. doi: 10.1104/pp.15.00353

Ying S, Zhang DF, Fu J, Shi YS, Song YC, Wang TY (2012) Cloning and characterization of a maize bZIP transcription factor, ZmbZIP72, confers drought and salt tolerance in transgenic Arabidopsis. Planta 235(2), 253-266. doi: 10.1007/s00425-011-1496-7

Yu YC, Wang L, Chen JC, Liu ZH, Park CM, Xiang FN (2017) WRKY71 acts antagonistically against salt-delayed flowering in Arabidopsis thaliana. Plant Cell Physiol 59(2), 414-422. doi: 10.1093/pcp/pcx201

Zhang DP, Wang YZ, Shen JY, Yin JF, Li DH, Gao Y, Xu WF, Liang JS (2018) OsRACK1A, encodes a circadian clock-regulated WD40 protein, negatively affect salt tolerance in rice. Rice 11(1). doi: 10.1186/s12284-018-0232-3

Zhao H, Jiang J, Li KL, Liu GF, Tsai CJ (2017) Populus simonii × Populus nigra WRKY70 is involved in salt stress and leaf blight disease responses. Tree Physiol 37(6), 1-18. doi: 10.1093/treephys/tpx020

\section{Supplementary Material Legends}

Supplementary Figure S1 Venn diagram of the number of DEGs in the leaves and roots of Z004 and Z011 after salt treatments (a) Venn diagram of the number of DEGs in Z0041L vs Z0040L, Z00424L vs Z0040L, and Z00472L vs Z0040L. (b) Venn diagram of the number of DEGs in Z0041R vs Z0040R, Z00424R vs Z0040R, and Z00472R vs Z0040R. (c) Venn diagram of the number of DEGs in Z0111L vs Z0110L, Z01124L vs Z0110L, and Z01172L vs Z0110L. (d) Venn diagram of the number of DEGs in Z0111R vs Z0110R, Z01124R vs Z0110R, and Z01172R vs Z0110R

Supplementary Figure S2 qRT-PCR validation of 20 genes randomly selected from the 39 DEGs in Table 2 in Z004 and Z011 roots. The error bars indicate SE

Online Resource 1 The proportion of reads in the Z. japonica genomic exon, intron and intergenic regions

Online Resource 2 Significant GO classification of DEGs in each comparison

Online Resource 3 Pathway classification of the genes differentially transcribed in each comparison

Online Resource 4141 DEGs selected from each comparison of Z004 and Z011 roots

Online Resource 5 Primer sequences for qRT-PCR

\section{Figures}


(A)

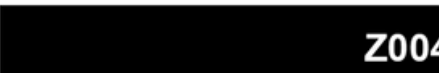

CK

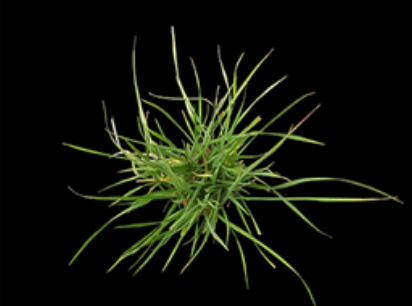

Z004

$350 \mathrm{mM} \mathrm{NaCl}$

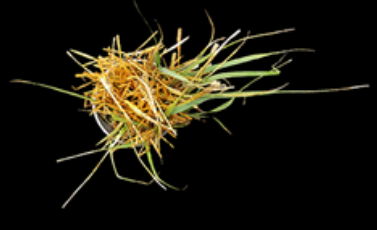

\section{Z011}

\section{$350 \mathrm{mM} \mathrm{NaCl}$}

CK

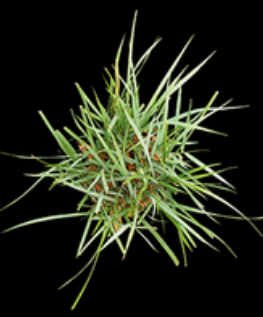

(B)

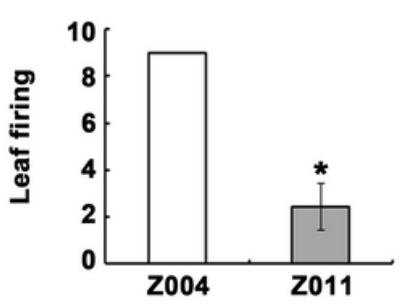

(C)

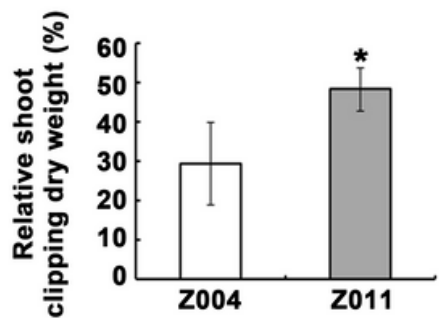

(D)

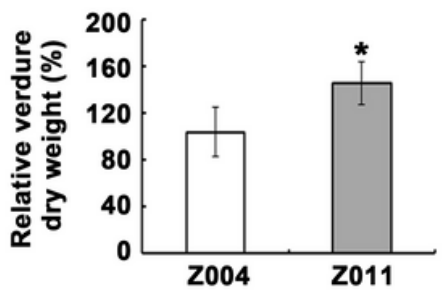

(E)

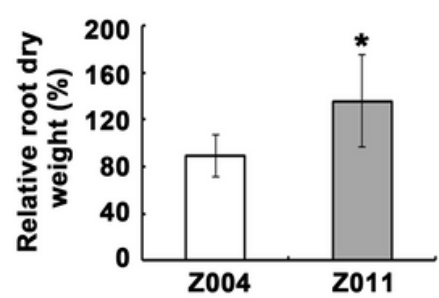

Figure 1

Phenotypic response of Z. japonica Steud. to salt stress (a) Two materials with contrasting salt tolerances, Z004 (salt sensitive) and Z011 (salt tolerant), were exposed to $350 \mathrm{mM} \mathrm{NaCl}$ for 40 days. (b) The leaf firings of Z004 and Z011 grasses after $\mathrm{NaCl}$ treatment for 40 days. (c) The relative shoot clipping dry weights of Z004 and Z011 after $\mathrm{NaCl}$ treatment for 40 days. (d) The relative verdure dry weights of Z004 and Z011 after $\mathrm{NaCl}$ treatment for 40 days. (e) The relative root dry weights of $\mathrm{Z} 004$ and $\mathrm{Z} 011$ after $\mathrm{NaCl}$ treatment for 40 days. Values are presented as the mean $\pm \mathrm{SE}$. Asterisks above the bars indicate significant differences between the respective values $(p<0.05)$ 
(A)

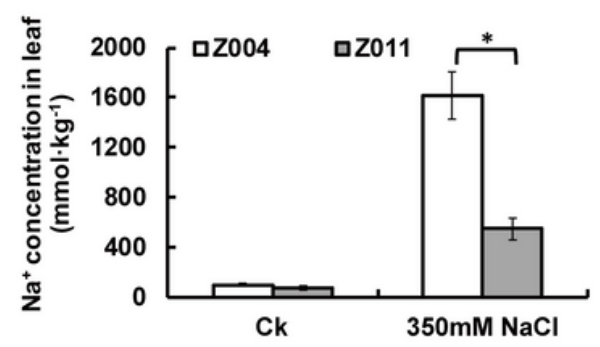

(D)

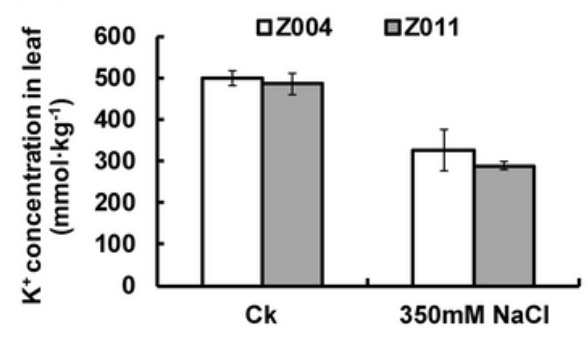

(B)

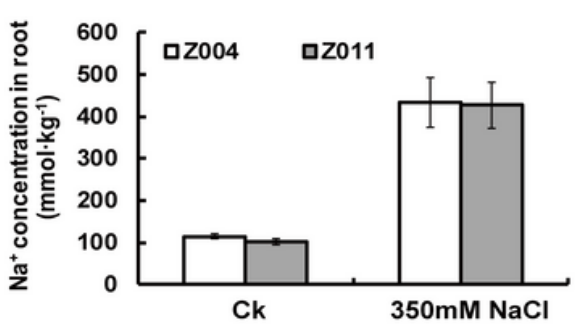

(E)

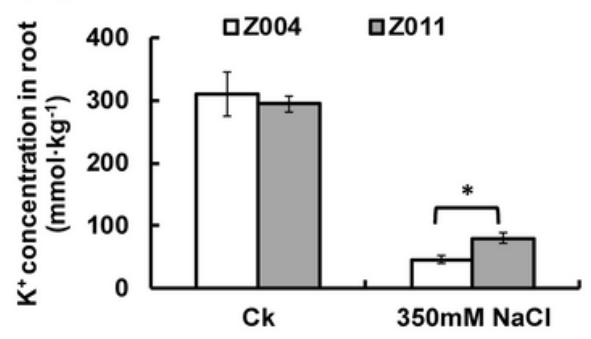

(C)

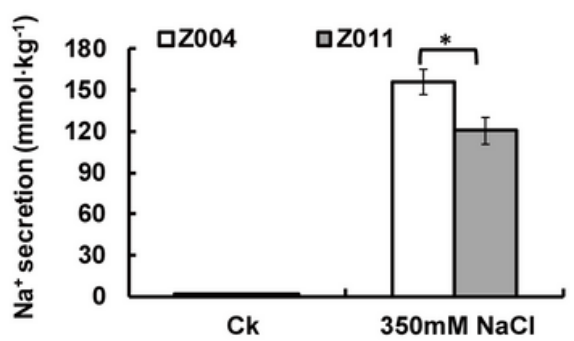

(F)

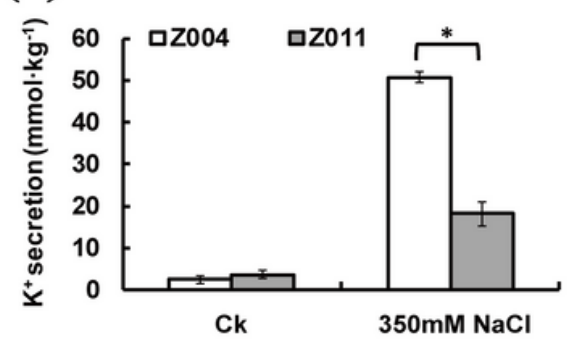

(G)

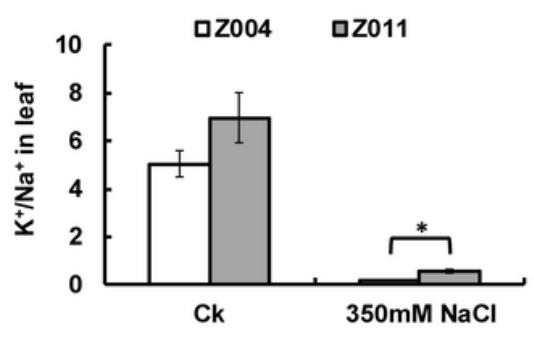

(H)

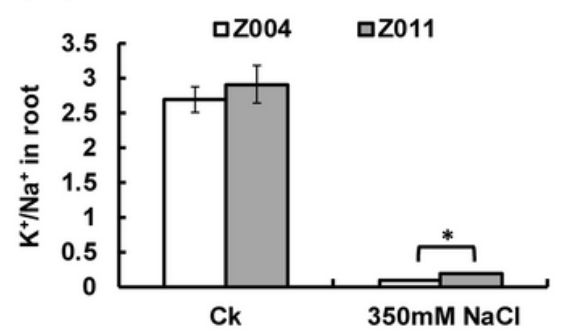

Figure 2

Physiological response of Z. japonica Steud. to salt stress (a) The Na+ concentration in Z004 and Z011 leaves after Ck and NaCl treatment. (b) The Na+ concentration in Z004 and Z011 roots after $\mathrm{Ck}$ and $\mathrm{NaCl}$ treatment. (c) Na+ secretion by Z004 and Z011 after Ck and NaCl treatment. (d) The K+ concentration in Z004 and Z011 leaves after $\mathrm{Ck}$ and $\mathrm{NaCl}$ treatment. (e) The $\mathrm{K}+$ concentration in $\mathrm{Z} 004$ and $\mathrm{Z} 011$ roots after $\mathrm{Ck}$ and $\mathrm{NaCl}$ treatment. (f) $\mathrm{K}+$ secretion by Z004 and Z011 after $\mathrm{Ck}$ and $\mathrm{NaCl}$ treatment. (g) The $\mathrm{K}+/ \mathrm{Na}+$ ratio in Z004 and Z011 leaves after $\mathrm{Ck}$ and $\mathrm{NaCl}$ treatment. (h) The $\mathrm{K}+/ \mathrm{Na}+$ ratio in Z004 and Z011 roots after $\mathrm{Ck}$ and $\mathrm{NaCl}$ treatment. Values are presented as the mean $\pm \mathrm{SE}$. Asterisks above the bars indicate significant differences between the respective values $(p<0.05)$

(A)

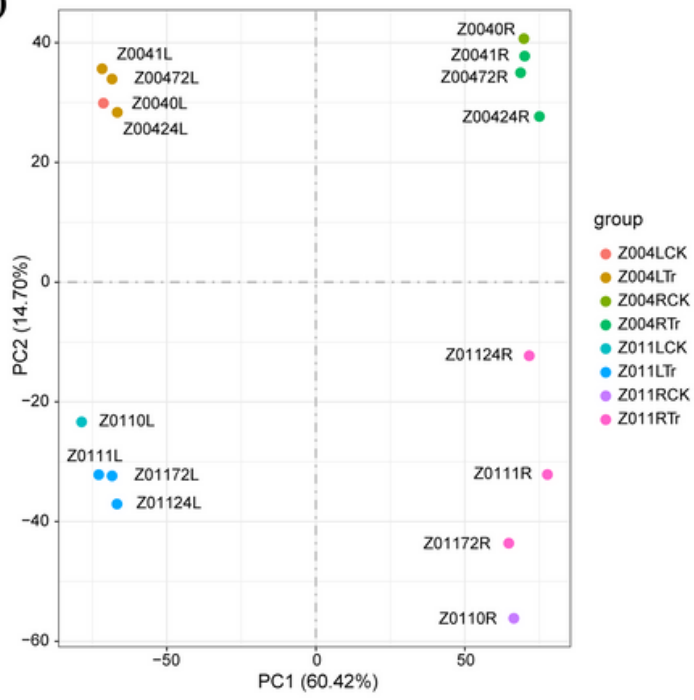

(B)

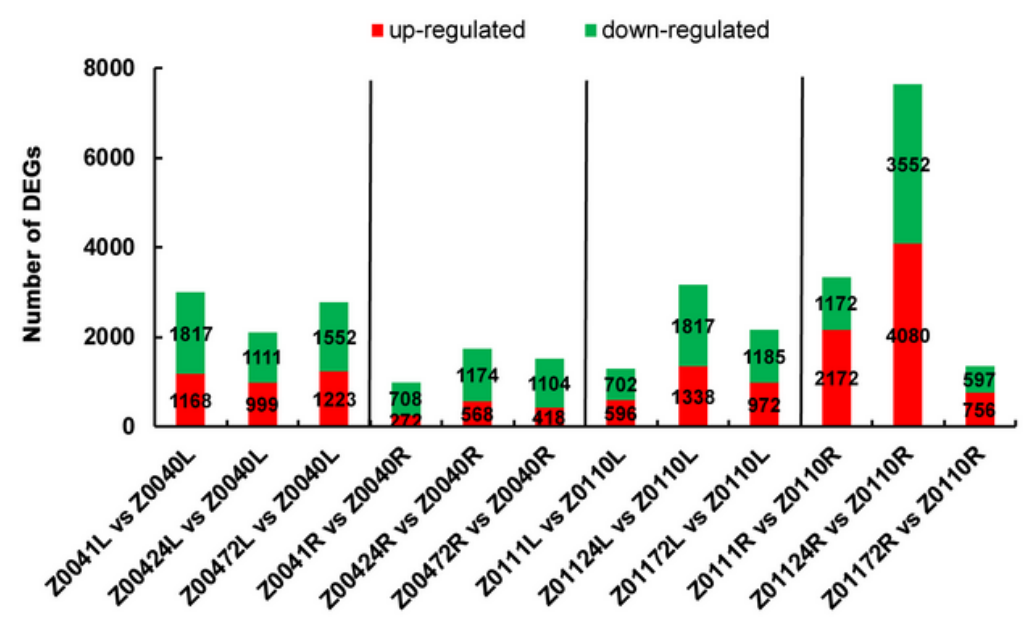


Figure 3

Z. japonica salt tolerance-related transcript expression profiles (a) PCA analysis of 16 transcriptome samples including four time points ( $0 \mathrm{~h}, 1 \mathrm{~h}, 24 \mathrm{~h}$, and $72 \mathrm{~h}$ ) and two tissues (leaves and roots) of Z004 and Z011 under salt treatments. The 0-h time point is the control group (CK), and the 1-h, 24-h and 72-h time points are the treatment group (Tr). (b) The number of up- or down-regulated DEGs in Z004 leaves (Z0041L vs Z0040L, Z00424L vs Z0040L, Z00472L vs Z0040L), Z004 roots (Z0041R vs Z0040R, Z00424R vs Z0040R, Z00472R vs Z0040R), Z011 leaves (Z0111L vs Z0110L, Z01124L vs Z0110L, Z01172L vs Z0110L) and Z011 roots (Z0111R vs Z0110R, Z01124R vs Z0110R, Z01172R vs Z0110R)

(A)

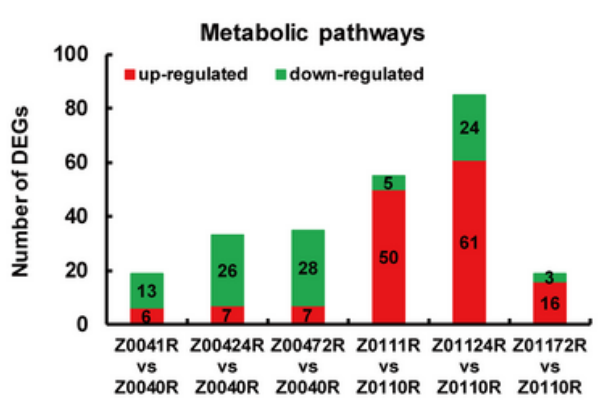

(B)

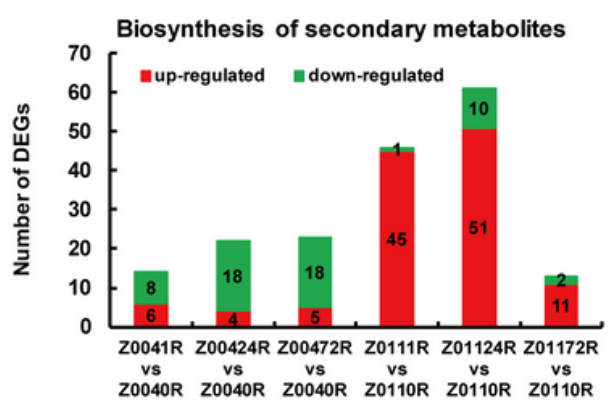

(C)

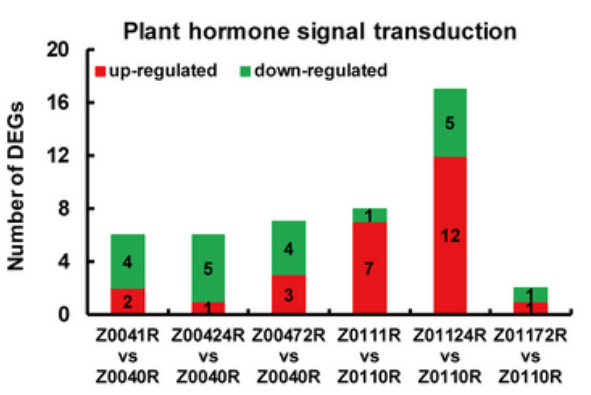

(D)

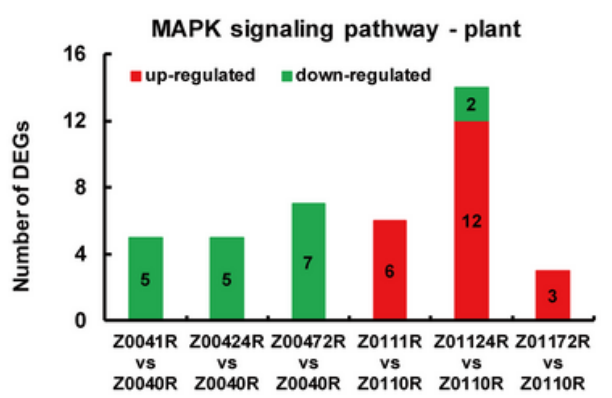

(E)

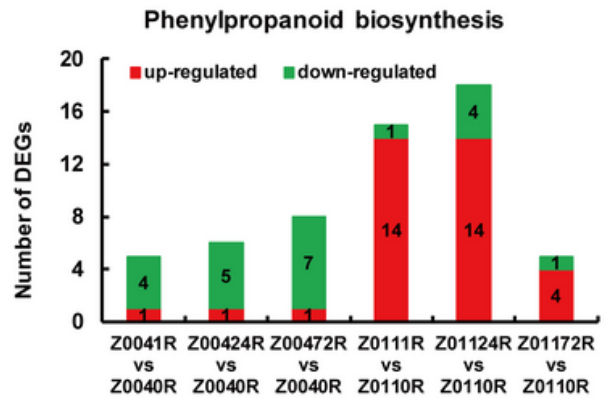

Figure 4

The number of up- or down-regulated DEGs in five KEGG pathways in Z004 roots and Z011 roots (a) The number of up- or down-regulated DEGs in metabolic pathways in Z0041R vs Z0040R, Z00424R vs Z0040R, Z00472R vs Z0040R, Z0111R vs Z0110R, Z01124R vs Z0110R and Z01172R vs Z0110R. (b) The number of up- or down-regulated DEGs in the biosynthesis of secondary metabolites in Z0041R vs Z0040R, Z00424R vs Z0040R, Z00472R vs Z0040R, Z0111R vs Z0110R, Z01124R vs Z0110R and Z01172R vs Z0110R. (c) The number of up- or down-regulated DEGs in plant hormone signal transduction in Z0041R vs Z0040R, Z00424R vs Z0040R, Z00472R vs Z0040R, Z0111R vs Z0110R, Z01124R vs Z0110R and Z01172R vs Z0110R. (d) The number of up- or down-regulated DEGs in the MAPK signaling pathway - plant in Z0041R vs Z0040R, Z00424R vs Z0040R, Z00472R vs Z0040R, Z0111R vs Z0110R, Z01124R vs Z0110R and Z01172R vs Z0110R. (e) The number of up- or down-regulated DEGs in phenylpropanoid biosynthesis in Z0041R vs Z0040R, Z00424R vs Z0040R, Z00472R vs Z0040R, Z0111R vs Z0110R, Z01124R vs Z0110R and Z01172R vs Z0110R 

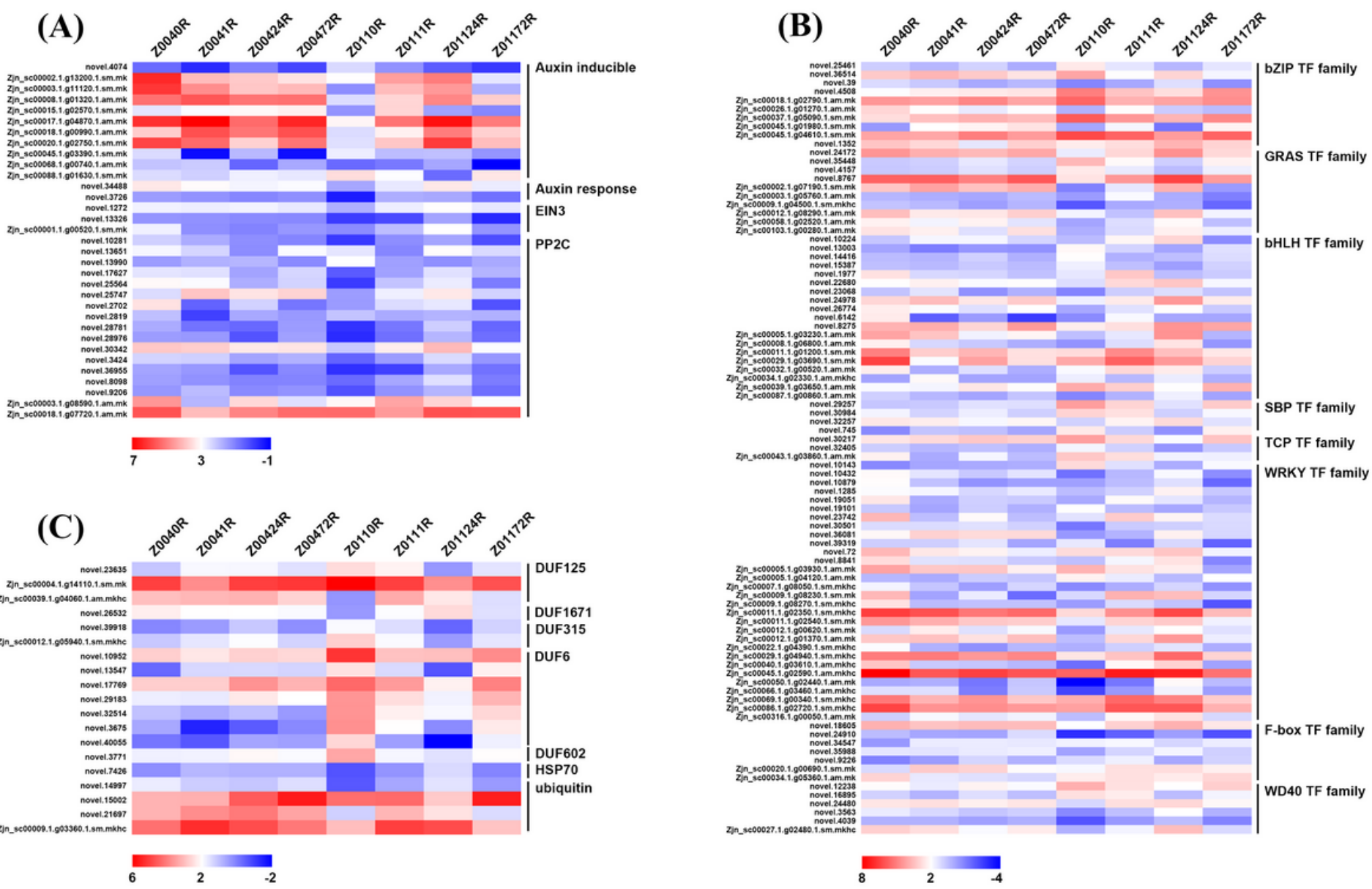

Figure 5

Heatmap of 141 DEGs in the roots of Z004 and Z011 at the four time points after salt treatments. The 141 DEGs are grouped into 3 main categories, and the red and blue rectangles represent the scale of the expression levels of each gene (log2FPKM). Red rectangles represent upregulated genes, and blue rectangles represent down-regulated genes

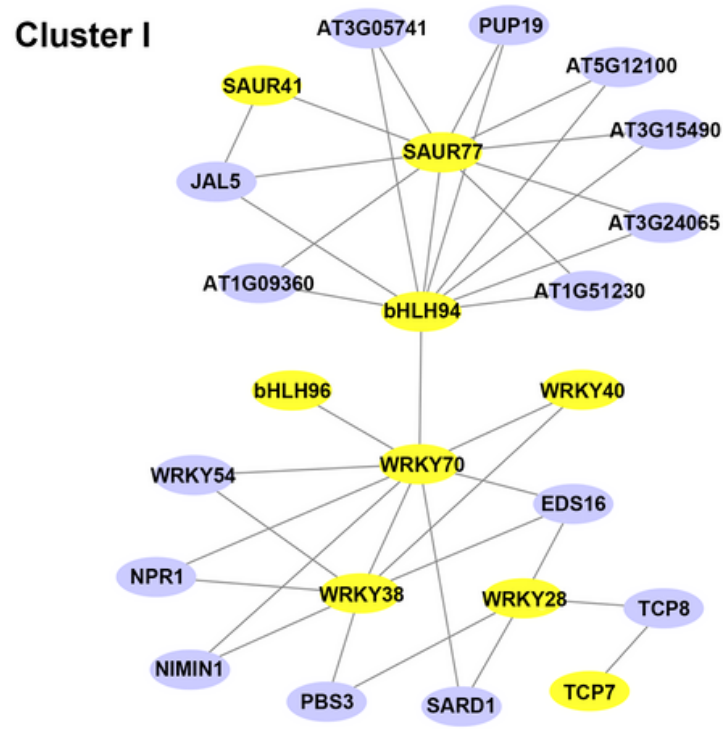

Cluster II

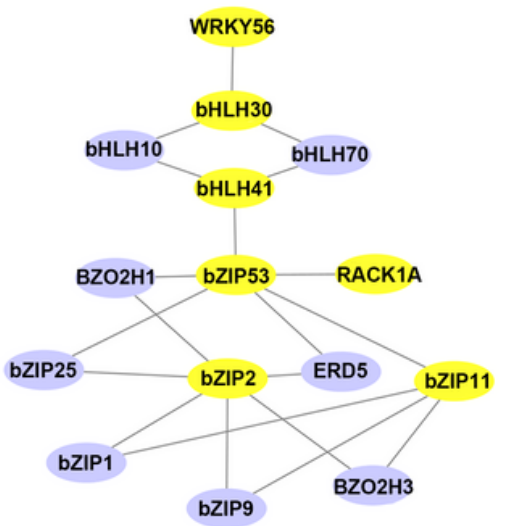

Cluster III

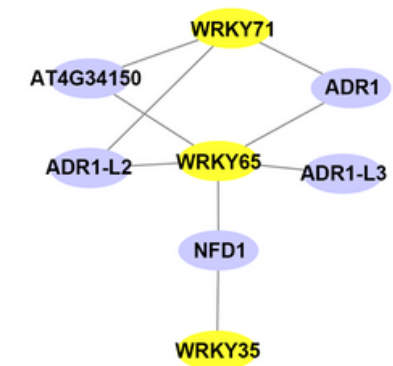

Figure 6

Protein interaction analysis of 39 significantly DEGs in Arabidopsis might reveal probable regulatory networks involved in Zoysia salt tolerance 


\section{Supplementary Files}

This is a list of supplementary files associated with this preprint. Click to download.

- Onlineresource4.xlsx

- Onlineresource5.pdf

- SuppplementaryfigureS1andfigures2.pdf

- Onlineresource3.xIsx

- Onlineresource2.xIsx

- Onlineresource1.pdf 